\title{
The serological response to SARS corona virus- 2 is characterized by frequent incomplete maturation of functional affinity (avidity)
}

Georg Bauer ( $\nabla$ georg.bauer@uniklinik-freiburg.de )

Universitaetsklinikum https://orcid.org/0000-0003-3134-3885

Friedhelm Struck

Mikrogen $\mathrm{GmbH}$

Patrick Schreiner

Mikrogen $\mathrm{GmbH}$

Eva Staschik

Mikrogen $\mathrm{GmbH}$

Erwin Soutschek

Mikrogen $\mathrm{GmbH}$

Manfred Motz

Mikrogen $\mathrm{GmbH}$

Article

Keywords: SARS CoV-2 , humoral immune systems, IgG, avidity

Posted Date: November 23rd, 2020

DOI: https://doi.org/10.21203/rs.3.rs-104847/v1

License: (9) (i) This work is licensed under a Creative Commons Attribution 4.0 International License.

Read Full License 
The serological response to SARS corona virus-2 is characterized by frequent incomplete maturation of functional affinity (avidity)

\author{
Georg Bauer ${ }^{1,2,4^{*}}$, Friedhelm Struck ${ }^{3}$, Patrick Schreiner ${ }^{3}$, Eva Staschik ${ }^{3}$, \\ Erwin Soutschek ${ }^{3}$, Manfred Motz ${ }^{3}$
}

1) Institute of Virology, Medical Center - University of Freiburg

2) Faculty of Medicine, University of Freiburg, Freiburg, Germany

3) Mikrogen GmbH, Neuried, Germany

4) Address all correspondence to:

Prof. Georg Bauer, Ph.D.

Institute of Virology,

Hermann-Herder Str. 11,

D-79104 Freiburg, Germany

Email: georg.bauer@uniklinik-freiburg.de 
The humoral immune systems controls viral infections through recognition of critical viral target structures, selective proliferation stimulation of IgM-presenting B cells, class switch to IgG-generating B cells and subsequent affinity maturation of IgG. Affinity maturation is achieved through proliferation, hypermutation and clonal selection of IgG-generating B cells (1-4). The establishment of high affinity IgG is essential for sustained protective antiviral effects (5-10).

While analyzing the maturation of functional affinity (avidity) of IgG towards SARS CoV-2 nucleoprotein, surface protein and its receptor-binding domain, we realized that avidity maturation was frequently not completed after infection. This finding gives insight into the biological strategy of SARS CoV-2 and is important for serodiagnosis. Incomplete avidity maturation might explain the observed decline of the humoral response (11-14), allow for secondary SARS CoV-2 infections and prevent the establishment of herd immunity. Therefore, future immunization strategies should achieve the goal to induce neutralizing $\lg G$ of high avidity.

\section{Main part}

During the pandemic with SARS CoV-2, the detection of viral genomes by PCR recognizes infections. Supplementary SARS CoV-2-specific antibody tests are necessary for an assessment of infection past viremia, for epidemiology, health system management, and the implementation of immunization programs (15-18). However, a high degree of complexity of the serological response towards SARS CoV-2 has been recognized (19). It was not possible to discriminate between acute and past infections by differential determination of SARS CoV-2-specific IgM and $\lg G$, as there is no regular pattern of the occurrence of these immunoglobulins. As avidity maturation occurs unidirectionally and irreversibly, and has been proven to be 
crucial for the correct serodiagnosis of many types of viral infections $(3,20-29)$, it was suggested to establish avidity determination for SARS CoV-2 serology (19).

The analysis of the kinetics of IgG responses towards nucleoprotein (NP), surface protein-1 (S1) and the receptor-binding domain (RBD) of S1 protein after symptomatic SARS CoV-2 infections, using immunoblots, led to surprising results (Figure 1): 1) An initial rapid increase in the IgG concentration was followed by a plateau phase and a subsequent decline in some cases. This finding is in line with the reports by Strömer et al. $(12,13) .2)$ Parallel assays, in which urea treatment had been included (Figure $1 \mathrm{~A}, \mathrm{C} . \mathrm{E}$ ), showed that most of the antibodies were removed by $7 \mathrm{M}$ urea. They therefore seemed to be of relatively low avidity. The analysis of the avidity indices (Figure 1 B, D, F) showed that initially partially matured avidity was not further developed at later time points. These findings indicate that the lgGs towards NP, S1 and RBD of SARS CoV-2 seem not to complete their avidity maturation, despite an initial onset. This breakpoint of interrupted avidity maturation obviously correlated with the point of decrease in IgG production. Incomplete avidity maturation is quite distinct from the regular pattern of completed avidity maturation in other viral systems $(3,19,20-29)$. For example, treatment with $7 \mathrm{M}$ urea allows a clear-cut distinction between acute and past Epstein-Barr virus infections based on low or high avidity of Anti-EBV-IgG (Suppl. Figure 1).

Parallel determination of the avidity indices of $\lg G$ directed towards seasonal coronavirus in one of our samples showed constant values that were either high or low (Figure $1 \mathrm{E}, \mathrm{F}$ ). This result gave a first indication that the lack of avidity maturation can be found for corona viruses other than SARS CoV-2 as well. Figure 2 A summarizes the concentrations of antibodies directed towards the three SARS CoV-2 antigens. Obviously, there is a broad variation of the value of grey intensities 
over the whole time from which sera had been taken. There is no general deducible pattern of relative height of the responses versus the nature of the antigen. However, in all cases, even those with very low grey intensity values, antibody responses in each individual serum were always developed towards all three antigens tested.

Determination of avidity revealed quite low values of avidity indices during the first 30 days and a very moderate increase over time (Figure $2 \mathrm{~B}$ ). Reaching an avidity index of more than 0.6 was the exception and only found in rare cases in the time window between 50 and 90 days. These findings are in contrast to the findings for infection with other viruses, such as EBV, where low avidity of IgG is characteristic for the early phase of infection and is regularly followed by high avidity $(24,25,30-32$; see also Supplementary Figure 1). Importantly, avidity indices for IgG directed towards S1 and RBD of SARS CoV-2 seemed to be very similar in most samples, whereas there were marked differences in avidity between IgG towards these two markers and IgG directed towards NP.

These data confirm that the immune response towards SARS CoV-2 proteins shows an initial rise in avidity, reaching varying low to intermediate values, followed by cessation of further continuation of avidity maturation. A general low avidity of IgG towards SARS CoV-2 proteins has also been reported by other groups $(13,14,33$, 34) though these authors did not recognize the specific kinetics of avidity maturation with its initial onset, followed by abrogation of maturation. Figure 3 illustrates the abrogation of avidity maturation in a characteristic case. Between day 27 and day 34 , avidity was markedly increased for antibodies directed towards NP, RBD and S1, as seen by the shift of the titrations curves with varying concentrations of urea. Nevertheless, avidity determined with the sharply discriminating concentration of $7 \mathrm{M}$ urea was still considerably low. After additional 16 days (on day 50), avidity for IgG 
towards NP only showed a marginal increase, whereas there was no significant increase in avidity for IgG towards RBD and S1. These findings demonstrate that avidity maturation seemed to be abrogated around day 34 after onset of disease. Interestingly, this particular serum contained IgG toward NP of the seasonal coronaviruses 229E and NL63, of high or low avidity, respectively. There was no change in avidity of these antibodies between day 27 and day 50, indicating that the low avidity of anti-NL63 had been established and preserved even before infection with SARS CoV-2. This finding points to incomplete avidity maturation in some cases after infection with seasonal corona viruses.

A systematic look at the serology of the seasonal coronaviruses $229 \mathrm{E}, \mathrm{NL} 63$; OC43 and HUK1 revealed a) that abrogated avidity maturation of $\lg G$ towards this group seemed to occur as well, but less frequent as for SARS CoV-2 and b) that there was a marked difference between the $\lg G$ responses towards the different seasonal corona viruses (Supplementary Figure 6).

The comparison of the antibody concentrations and avidity indices of sera from patients with symptoms of disease without and with requirement for hospitalization showed that there was a trend for higher grey intensities and a marked increase in avidity in the hospitalized patients (Figure 4). The relative increase in the number of sera reaching grey intensity values higher than 500 for hospitalized patients was paralleled by a relative decrease in the number of sera with grey intensity values below 200 units. Though both patient groups showed examples of immature avidity (avidity index $<0.3$ ) even more than one month past the onset of disease, the percentage of partially mature $\lg G$ (avidity indices between 0.3 and 0.6 ) had the tendency to be higher in the hospitalized patients compared to control patients 
(Figure $4 \mathrm{~B})$. Moreover, patients with IgG of high avidity $(>0.6)$ were only found in the hospitalized group. These data show that at least in a low percentage of patients, one of the outcomes of severe illness due to COVID-19 is the final establishment of anti-SARS-CoV-2 IgG of high avidity. This feature correlates with the finding that the humoral immune response is often higher and more stable in patients with severe disease compared to control patients that only developed mild symptoms (11). It is also in line with the findings by Benner et al. (33), who reported on a relative increase in avidity of IgG towards SARS CoV-2 in hospitalized patients.

Due to the mechanism that is underlying affinity/avidity maturation, a sustained and optimal availability of target antigen is necessary to ensure complete affinity maturation $(9,35-37)$. It seems that neutralizing IgG of low affinity is sufficient in the case of SARS CoV-2 and other corona viruses to interfere with the low viremia due to localized infection. As a result, maturation of affinity might be abrogated due to lack of antigen, with the consequence of breakdown of IgG production and lack of further affinity maturation and generation of memory B cells.

In the case of more severe disease due to SARS CoV-2, the initial control of virus might be less effective, with the consequence of enhanced viremia and resultant more severe disease. Successful counteraction by the humoral immune system then might stop viral replication. Due to higher viral load, and therefore better supply of the immune system with for longer time, the resultant affinity maturation might be be more pronounced. As a consequence, protection towards reinfection at a later time point is likely to be more efficient. The findings of a strict correlation between neutralizing potential of IgG directed towards SARS CoV-2 S1 protein/RBD and its avidity $(33,34)$ is supporting this idea, though the overall avidity did not reach high values. 
Taken together, these findings allow to predict that successful protective immunization towards SARS CoV-2 might depend on complete affinity (avidity) maturation. This goal can only be achieved if the immunogenic surface proteins will be available for the immune system at sufficiently high concentrations and for sufficiently long times. It seems desirable that immunization leads to a higher degree of avidity maturation than natural infection with SARS CoV-2. The control of avidity maturation of IgG towards S1 or preferentially RBD of SARS CoV-2 after immunization therefore might give essential information on protection. If these predictions hold, we propose to include avidity determination into the establishment of vaccination programs towards SARS CoV-2. 


\section{Methods}

\section{Sera.}

\section{SARS-CoV-2-positive sera:}

A. Sera from adult outpatients with clinical signs of COVID-19 and SARS CoV-2 infection confirmed by PCR were collected after a call in the Munich area for voluntary donation of a serum sample for serological analysis (SARS CoV-2 and saisonal corona viruses). The samples were drawn by the respective family doctors. The volunteers explicitely gave their written consent for testing. The group of logistic support of Mikrogen $\mathrm{GmbH}$ collected the sera, personal data as well as information related to the sample and on clinical symptoms.

The samples were then anonymized and given to the group of Research and Development of Mikrogen $\mathrm{GmbH}$ for professional testing in the newly established recomLineSARS CoV-2 line assay, including avidity testing. For the group of Research and Development and for the the first author (G.B.) who analyzed the data, no personal data were available and the information was restricted to the clinical symptoms of the patients, the data of extraction of the sera and the time between onset of clinical symptoms and extraction of the sera.

B. Sera from adult hospitalized patients with COVID-19 and SARS CoV-2 infection confirmed by positive PCR, together with information on the time of onset of clinical symptoms were kindly provided by Prof. P. Luppa, Technical University Munich. 14/40 hospitalized patients required intensive care.

\section{SARS CoV-2-negative sera:}

300 anonymized plasma samples from healthy adult blood donors were purchased from the Bavarian Red Cross. The blood donor sera were collected before the 
outbreak of the SARS-CoV-2 pandemic, i. e. before November 2019. They have been assayed to determine the specificity of the recomLine SARS-CoV-2.

Sera were stored at $-20^{\circ} \mathrm{C}$ until they were tested in the immunoassays.

\section{Immunoblot assay:}

A. Production of recomLine SARS-CoV-2 nitrocellulose strips: Individual concentrations of purified recombinant antigens NP, RBD, S1 of SARS-CoV-2, as well as NP of 229E, NL63, OC43, HKU1 were applied directly onto nitrocellulose membranes in separate lanes. Production was standardized and the resultant strips were evaluated (see below for details), resulting in product \# 7374 of Mikrogen $\mathrm{GmbH}$. The assay has been CE-marked.

B. Procedure of the line immunoassay: The reactivity of $1: 100$ dilutions of serum antibodies against the recombinant antigens was detected with peroxidase-labelled anti-human IgG antibody and the use of precipitating tetramethylbenzidine. The first incubation of serum and test strips was for $1 \mathrm{~h}$, followed by three washing steps with buffer. The incubation of the strips with peroxidase-labelled anti-human $\lg G$ antibody was for $45 \mathrm{~min}$, followed by three washing steps. Treatment with tetramethylbenzidine was for 8 min.

The line immunoassays were carried out in a semiautomatic processor Dynablot (Dynex Technologies GmbH, Denkendorf, Germany) with manual serum pipetting according to instruction manual provided by Mikrogen $\mathrm{GmbH}$. An Epson J371A scanner (Epson, Japan) and recomScan software (Mikrogen GmbH, Neuried, Germany) were used according to the instruction manuals. 
C. Avidity determination: sera were incubated for $1 \mathrm{~h}$ with the recomLine SARS-CoV2 test strips in duplicate; then both replicates incubated for 5 min with wash buffer, and one assay was incubated in wash solution, while the parallel assay replicate was treated with defined concentrations of urea for 3 minutes; after 3 additional washing steps both assay replicates were processed with anti-human IgG antibody labelled with peroxidase and detected as outlined above to describe the line immunoassay procedure. The grey intensity area output by recomScan on the urea treated test strip was divided by the grey intensity of the parallel assay replicate to determine the avidity index arithmetically. Standard urea treatment for sharp distinction between IgGs of low and high avidity was $7 \mathrm{M}$. Where indicated, other concentrations of urea were used during our analysis.

\section{Precision and reproducibility of the immunoassay}

D.1 Intra-lot (within-run) precision. Intra-lot (within run) precision was determined with one lot, of test strips, one dynablot processor, 2 operators with one experiment each on the same day. 4 IgG-positive specimen were tested 10 or 11 times each in one experiment in recomLine SARS-CoV-2, respectively. Variation coefficient of positive sera or sera above 25 grey intensities was below $<19.9 \%$

NP_HKU1 and NP_OC43 were also included in the data set and comply with the specification above ( $\mathrm{Vc}$ below $\leq 20 \%)$.

D2.Intra-Lot (between-day) precision. Intra-Lot (between-day) precision was determined for a panel of 4 samples (high positive, low positive, greyzone, negative). The serea were assayed in one lot as 4 fold replicates, per day, assayed on 3 different days, assayed by 2 operators, one lot of test kit, 2 dynablot processors. The variation coefficient of positive sera or sera above 50 grey scales was $<21.5 \%$ 
D3.Interlot (between-day) precision. Interlot (between-day) precision was determined with a panel of 4 samples (high positive, low positive, greyzone, negative) on 3 different days (3 lots of test kits, 2 dynablot processors) as 4 fold replicates per day, assayed by 2 operators. The variation coefficient of positive sera or sera above 50 grey intensities was $<18 \%$.

\section{E. Diagnostic sensitivity and specificity of the immunoassay}

Sensitivity of the recomLineSARS CoV-2 assay was determined using the sera from 54 patients with PCR-confirmed SARS CoV-2 infection. Sensitivity was $85.7 \%$ for the time span $<12$ days after onset of clinical symptoms, $95.2 \%$ for the time span between 12 and 23 days and $100 \%$ for sera taken later than 23 days after the onset of clinical symptoms.

Specificity of the recomLineSARS CoV-2 assay was determined using a) 300 sera from healthy blood donors (taken before the pandemics), b) 191 sera with potential crossreactivity due to infection with seasonal corona viruses, respiratory syncytial virus, influenza A and B, adenovirus, mycoplasma, chlamydia, EBV (IgM-positive), CMV (IgM-positive), anticellular reactivity (ANA/ENA) or pregnancy, c) 78 sera with possible interfering potential (lipemic, icteric, hemolytic sera and sera containing rheumatoid factor). The determined specificities were $99,7 \%$ for $a, 97,9 \%$ for $b$ and $97,5 \%$ for c.

\section{Statistics}

Due to the established professional performance of the recomLine SARS CoV-2 line assay (as shown above), all determinations were performed under conditions of routine diagnostics, i. e. sera were tested individually in single assays. Three sera were tested in repeat experiments, using variable concentrations of urea both in the 
initial and the repeat experiment. No statistical significant difference was observed between the initial and the repeat experiment.

In the experiments described in Figures 1,2 and 4 measurements were taken from distinct samples. In the experiment described in Figure 3 the three distinct samples were tested repeatedly under varying conditions of urea treatment.

The data analysis by G. Bauer was performed on the basis of raw data.

The Yates continuity corrected chi-square test (two-sided) was used for the statistical determination of significances $(p<0.01=$ significant; $p<0.001=$ highly significant $)$. 


\section{Acknowledgements}

We thank Dr. P. Luppa (Technical University Munich) and many voluntary donors for providing sera for this study. We thank Dr. S. Schulz (Mikrogen $\mathrm{GmbH}$ ) for professional logistic support.

\section{Authors contributions:}

Friedhelm Struck, Patrick Schreiner, Erwin Soutschek and Manfred Motz: Development and evaluation of the test system; organizing and supervising testing in-house; documentation and discussion of data; commenting and correcting the manuscript. Georg Bauer: Analysis of raw data; Generation of the graphs; Conceptualization; writing the manuscript.

\section{Conflict of interests statements}

E. Soutschek and M. Motz are owners of Mikrogen GmbH. E. Soutschek is the present CEO of Mikrogen GmbH. F. Struck, E. Stachik and P. Schreiner are employees of Mikrogen $\mathrm{GmbH}$.

The determination of avidity of antibodies, using immunoblots or other techniques that allow the parallel measurement of humoral immune reactions towards several antigens in one assay, has been patented by Mikrogen GmbH (WO 00/54055; PCT/EP00/01883). In addition, a new patent application for a method to determine the antibodies towards SARS CoV-2 has been filed by Mikrogen $\mathrm{GmbH}$ and is pending (EP 2019/2550). Mikrogen $\mathrm{GmbH}$ develops and produces test systems for serological analysis of infectious diseases.

G. Bauer is a member of the Medical Faculty of the University of Freiburg. He is the inventor of WO 00/54055; PCT/EP00/01883 and one of the coinventors of EP 2019/2550.

\section{Data availability statement}

All raw data used for this study are presented within the study as "Grey intensity units".

\section{Code availability statement}

This study does not use custom code or mathemathical algorithms. 


\section{References}

1 Eisen HN, Siskind GW. Variations in the affinities of antibodies during the immune response. Biochemistry 1964; 3: 996-1008.

2 Foote J, Milstein C. Kinetic maturation of an immune response. Nature 1991; 352: 530-531.

3 Hedman K, Lappalainen M, Söderlund M, Hedman L. Avidity of IgG in serodiagnosis of infectious diseases. Rev Med Microbiol 1997; 4: 123-129.

4 Hazel SL. Clinical utility of avidity assays. Expert Opinion on medical diagnostics 1: 511519,2007

5 Junker AK, Tilley P. Varicella-zoster virus antibody avidity and subclass patterns in children with recurrent chickenpox. J Med Virol 1994; 43: 119-124.

6 Martin KA, Junker AK, Thomas EE, Van Allen MI, Friedman, JM. Occcurence of chickenpox during pregnancy in women seropositive for varicella-zoster virus. J Infect Dis 1994; 170: 991-995.

7 Boppana SB, Britt WJ. Antiviral antibody responses and intrauterine transmission after primary maternal cytomegalovirus infection. J Infect Dis 1995; 171: 1115-1121.

8 Delgado MF, Coviello S, Monsalvo AC, Melendi GA, Hernandez JZ, Batalle JP, Diaz L, Trento A, Chang H-Y, Mitzner W, Ravetch J, Melero JA, Irusta PM, Polack FP. Lack of antibody affinity maturation due to poor Toll-like receptor stimulation leads to enhanced respiratory syncytial virus disease. Nature Medicine, 2009; 15: 34-41.

DOI 10.1099/jgv.0.001439

9 Kontio M, Jokinen S, Paunio M, Peltola H, Davidkin I. Waning antibody levels and avidity: implications for MMR vaccine-induced protection. JID 2012; 206: 1542-1548.

10 Puschnik A, Lau L., Cromwell EA., Balmaseda A, Zompi S, Harris E. Correlation between Dengue-specific neutralizing antibodies and serum avidity in primary and secondary Dengue virus 3 natural infections in humans.PLOS Neglected Tropical Diseases, 2013; 7: e2274

11 Long Q-X, Tang X-J, Shi Q-L, Li Q, Deng H-J, Yuan J, Hu J-L, Xu W, Zhang Y, Lv F-J, Su K, Zhang F, Gong J, Wu B, Liu X-M, Li J-J, Qiu J-F, Chen J, Huang A-L. Clinical and immunological assessment of asymptomatic SARS-CoV-2 infections. Nature Med 2020. https://doi.org/10.1038/s41591-020-0965-6

12 Seow J, Graham C, Merrick B, Acors S, Steel KJA, Hemmings O, O'Bryne A, Kouphou N, Pickering S, Galao RP, Betancor G, Wilson HD, Signell AW, Winstone H, Kerridge C, Temperton N, Snell L, Bisnauthsing K, Moore A, Green A, Martinez L, Stokes B, Honey J, Barras Al, Arbane G, Patel A, O'Connell L, O'Hara G, MacMahon E, Douthwaite S, Nebbia G, Batra R, Martinez-Nunez R, . Edgeworth JD, Neil SJD, Malim MH, Doores KJ. Longitudinal evaluation and decline of antibody responses in SARS-CoV-2 infection. medRxiv preprint doi: https://doi.org/10.1101/2020.07.09.20148429 
13 Strömer A, Grobe O, Rose R, Fickenscher H, Lorentz T, Krumbholz A. Diagnostic accuracy of six commercial SARS-CoV-2 lgG/total antibody assays and identification of SARS-CoV-2 neutralizing antibodies in convalescent sera. medRxiv doi: https://doi.org/10.1101/2020.06.15.20131672

14 Strömer A, Rose R, Grobe O, Neumann F, Fickenscher H, Lorentz T, Krumbholz A. Kinetics of nucleo- and spike protein-specific immunoglobulin $\mathrm{G}$ and of virus-neutralizing antibodies after SARS-CoV-2 infection. Microorganisms 2020, 8, 1572;

doi: 10.3390/microorganisms8101572

15 Zhao J, Yuan Q, Wang H, Liu W, Liao X, Su Y, Wang X, Yuan J, Li T, Li J; Qian S, Hong C, Wang F, Liu Y, Wang Z, He Q, Li Z, He B, Zhang T, Fu Y, Ge S, Lei Liu L, Zhang J, Xia N, Zhang Z. Antibody responses to SARS-CoV-2 in patients of novel coronavirus disease 2019 Clinical Infectious Diseases, 2020, https://doi.org/10.1093/cid/ciaa344

16 Jin Y, Wang M, Zuo Z, Fan C, Ye F, Cai Z, Wang Y, Cui H, Pan K, Xu A. Diagnostic value and dynamic variance of serum antibody in coronavirus disease. International Journal of Infectious Diseases 94 (2020) 49-52

17 Xiao S-Y, Wu Y, Liu H. Evolving status of the 2019 novel coronavirus infection: Proposal of conventional serologic assays for disease diagnosis and infection monitoring. J Med Virol 2020; 92:464-467.

18 Wölfel R, Corman VM, Guggemos W, Seilmaier M, Zange S, Müller MA, Niemeyer D, Jones TC, Vollmar P, Rothe C, Hoelscher M, Bleicker T, Brünink S, Schneider S, Ehmann R, Zwirglmaier K, Drosten C, Wendtner C.Virological assessment of hospitalized patients with COVID-2019. Nature 2020; https://doi.org/10.1038/s41586020-2196-x (2020).

19 Bauer G. The variability of the serological response to SARS-corona virus-2: Potential resolution of ambiguity through determination of avidity (functional affinity). J Med Virol. 2020;1-12.https://doi.org/10.1002/jmv.26262

20 Hedman K, Rousseau SA. Measurement of avidity of specific lgG for verification of recent primary rubella. J Med Virol 1989; 27: 288-292. doi.org/10.1002/jmv.1890270406

21 Hedman K, Seppalä I. Recent rubella infection indicated by a low avidity of specific IgG. J Clin Immunol 1988; 8: 214-221.

22 Robertson P, Beynon S, Whybin R, Brennan C, Vollmer-Conna U, Hickie I, Lloyd A. Measurement of EBV-lgG anti-VCA avidity aids the early and reliable diagnosis of primary EBV infection. J Med Virol 2003; 70:617-623.

23 Gassmann C, Bauer G. Avidity determination of IgG directed against tick-borne encephalitis virus improves detection of current infections. J Med Virol 1997; 51: 242-251.

24 Bauer G. Simplicity through complexity: immunoblots with recombinant antigens as the new gold standard in Epstein Barr virus serology. Clin. Lab. 47: 223-230, 2001. 
25 Niller HH, Bauer G. Epstein-Barr Virus: Clinical Diagnostics. Meth Mol. Biol 2017; 1532: 33-55.

26 Lazzarotto T, Varani S, Spezzacatena P, Gabriell L, Pradelli P, Guerra B, Landini MP. Maternal IgG Avidity and IgM Detected by Blot as Diagnostic Tools to Identify Pregnant Women at Risk of Transmitting Cytomegalovirus. Virol Immunol 13: 137-141, 2009. https://doi.org/10.1089/vim.2000.13.137

27 Ward KN, Turner DJ, Couto Parada X, Thiruchelvam AD. Use of immunoglobulin G antibody avidity for differentiation of primary human herpesvirus 6 and 7 infections. J Clin Microbiol 2001; 39: 959-963. DOI: 10.1128/JCM.39.3.959-963.2001

28 Söderlund M, Brown C, Cohen BJ, Hedman K. Accurate serodiagnosis of B 19 parvovirus infections by measurement of IgG avidity. JID 1995; 171: 710-713.

29 Paunio M, Hedman K, Davidkin I, Peltola H. IgG avidity to distinguish secondary from primary measles vaccination failures: prospects for a more effective global measles elimination strategy. Exp Opininion Pharmacotherapy 2005; 4: 1215-1225.

30 Schubert J, Zens W, Weissbrich B. Comparative evaluation of the use of immunoblots and of $\lg G$ avidity assays as confirmatory tests for the diagnosis of acute EBV infections. $J$ Clin Virol 1998; 11: 161-172. Doi.org/10.1016/S0928-0197(98)00061-0

31 Pottgiesser T, Schumacher $\mathrm{Y}$ O, Wolfarth B, Schmidt-Trucksäss A and Bauer G. Longitudinal observation of Epstein-Barr virus antibodies in athletes and controls during a competition season. J. Med. Virol. 84: 1415-1422, 2012.

32 Pottgiesser T, Wolfarth B, Schumacher YO and Bauer G. Epstein-Barr virus serostatus: no difference despite aberrant patterns in athletes and control groups. Med \& Science in Sports and Exercise 38: 1782-1791, 2006.

33 Benner S, Patel EU, Laeyendecker O, Pekosz A, Littlefield K, Eby Y, Fernandez RE, Miller J, Kirby CS, Keruly M, Klock E, Baker OR, Schmidt HA, Shrestha R, Burgess I, Bonny TS, Clarke W, Caturegli P, Sullivan D, Shoham S, Quinn TC, Bloch EM, Casadevall A, Tobian AAR, Redd AD. SARS-CoV-2 antibody avidity responses in covid-19 patients and convalescent plasma donors. The Journal of Infectious Diseases, jiaa581, https://doi.org/10.1093/infdis/jiaa581

34 Klein SL, Pekosz A, Park H-S, Ursin RL, Shapiro JR, Benner SE, Littlefield K, Kumar S, Naik HM, Betenbaugh MJ, Shrestha R, Wu AJ, Hughes RM, Burgess I, Caturegli P, Laeyendecker O, Quinn TC, Sullivan D, Shoham S, Redd AD, Bloch EM, Casadevall A, Tobian AAR.Sex, age, and hospitalization drive antibody responses in 1 a COVID-19 convalescent plasma donor population. J Clin Invest. 2020.

https://doi.org/10.1172/JCl142004 
35 Brokstad KA, Cox RJ; Major D, Wood JM, Haaheim LR. Cross-reaction but no avidity change of the serum antibody response after influenza vaccination Vaccine 13, 1522-1528, 1995. https://doi.org/10.1016/0264-410X(95)00095-I

36 Kellam P, Barclay W. The dynamics of humoral immune responses following SARS-CoV2 infection and the potential for reinfection. Journal of General Virology, 2020.

37 French MA, Moodley Y. The role of SARS-CoV-2 antibodies in COVID-19:

Healing in most, harm at times. Respirology (2020) doi: 10.1111/resp.13852 


\section{Figure Legends}

Figure 1. Kinetics of the serological responses to SARS CoV-2 nucleoprotein (NP), receptor-binding domain (RBD) and surface protein $\mathrm{S} 1$.

Sera from three patients with COVID-19 confirmed by positive PCR and showing clinical symptoms were tested for IgG towards SARS CoV-2 NP, RBD and S1, as well as towards NP of the seasonal coronaviruses $229 \mathrm{E}, \mathrm{NL63}$; OC43 and HUK1 in the determination described under $E$ and $F$, using a recombinant line immunoassay. The immunoassays were performed without and with urea treatment $(7 \mathrm{M})$ for the determination of avidity. $A, C$, and $E$ shows the grey intensity values determined, whereas $B, D$, and $F$ show the calculated avidity indices.

The data show an initial increase of grey intensities for IgG towards the SARS CoV-2 proteins, which is followed by a plateau and even an indication of decline. Avidity maturation of the IgGs towards SARS CoV-2 is variable, but overall poor. The avidity index for IgG towards NP in B constantly stays at 0.1 , whereas the other two lgGs show initial marginal avidity maturation, ending at a plateau of 0.2 . The examples under $\mathrm{D}$ and $\mathrm{F}$ are characterized by an initial increase of avidity, followed by a plateau or only slight increase. Avidity of IgG towards seasonal coronaviruses was either of high avidity (D), or of high avidity towards NP of 229E and constantly low avidity towards NL63. These findings demonstrate that incomplete avidity maturation is characteristic for the response towards SARS CoV-2 and can also be found for other corona viruses.

Figure 2. IgG responses in 93 serum samples from patients with confirmed SARS CoV-2 infection and COVID-19. 93 sera from patients with clinical signs of Covid-19, quite predominantly showing ambulatory mild disease, and with positive SARS CoV-2 PCR test were tested with the recomline SARS-CoV-2 IgG test. The grey intensities $(A)$, which reflect the concentrations of the respective antibodies and the avidity indices (B) are shown in correlation to the time after onset of disease for each serum.

A. Reflectometric grey intensity values. The result shows a broad distribution of grey scales, which was similar at all time points. The percentages of sera showing values in the ranges $0-250,250-500$ and more than 500 grey intensity were not significantly different when sera taken before or after 50 days after onset of disease were compared, with the exception of IgG towards NP at less than 250 units. Importantly, all sera that gave a positive result showed positivity towards all three antigens tested, without exception.

In $93.5 \%$ of the sera with positive IgG towards S1, IgG towards RBD showed a value that was less than 20 grey intensity units different from that obtained for IgG S1, whereas in $6.5 \%$ of the sera, $\lg$ G values towards $S 1$ and RBD were different more than 20 grey intensity units $(p<0.001)$. In contrast, only $15 \%$ of sera showed IgG 
towards NP at a value that differed less than 20 grey intensity units from that of the respective IgG $S 1$ value and $84.9 \%$ showed higher difference $(p<0.001)$.

B. Avidity indices. Whereas the grey intensity values (indicative of the respective concentrations of $\operatorname{lgG}$ ) were broadly similar between sera taken within 50 days or after 50 days after onset of disease, the avidity of the $\mathrm{IgG}$ was increasing between these groups. However, it did not reach complete avidity maturation (as seen for a reference virus under Supplementary materials). 82.9 percent of the sera taken before day 50 showed an avidity index below 0.3 , whereas only $42.7 \%$ of the sera taken after 50 days showed similar low avidity $(\mathrm{p}<0.001)$. As an indication of partial avidity maturation, only $17.1 \%$ of sera taken before 50 days showed an avidity index between 0.3 and 0.6 and none an index above 0.6 , whereas $44 \%$ of the group taken after 50 days showed avidity indices between 0.3 and 0.6 , and $13.2 \%$ an index above 0.6 (indicative for nearly completed maturation) ( $p<0.001$ ). In summary, an overall low degree of avidity maturation was seen, with a minority of samples of high avidity at late time points.

Figure 3. Demonstration of the abrogation of avidity maturation.

Sera were available from a patient with Covid-19 and PCR-confirmed SARS CoV-2 infection taken at days 27, 34 and 50 after onset of disease. The sera were tested in avidity assays based on the recomLine SARS CoV-2 assay, with the modification that they remained either untreated with urea or were treated with the indicated concentrations of urea. This allows to titrate nominal avidity indices in relationship to the urea concentration. The shift of the resultant titration curves is indicative for avidity maturation. The figure shows that avidity for IgG towards NP (A) and RBD or S1 (B) had increased between day 27 and 34 ( $\mathrm{dt}=7$ days), but only showed minor (A) or no (B) further increase between days 34 and $50 \mathrm{dt}=16$ days). This finding is indicative for an abrogation of avidity maturation. Though the avidity indices for IgG towards RBD and S1 were higher compared to IgG towards NP, in none of the markers high avidity above 0.6 was reached. Remarkably, the avidity index of IgG towards the seasonal corona viruses 229E and NL63 either remained unchanged high (229E) or low (NL63) during the period of observation. This finding indicates that an incomplete avidity maturation can also be found for seasonal corona viruses.

Figure 4. The serological response towards SARS CoV-2 in Covid-19 patients without and with the need for hospitalization.

Sera from 37 patients with Covid-19 without need for hospitalization and 39 patients with need for hospitalization were taken within the first 50 days after onset of disease and tested in the recomLine SARS CoV-2 avidity assay.

A. Optical density. Relatively less hospitalized patients with moderate or severe disease showed grey intensity values in the OD range from $0-250$ grey intensity units compared to nonhospitalized patients showing ambulatory mild disease $(22.2 \%$ 
compared to $45 \% ; p<0.001$ ) and relatively more hospitalized patients showed grey intensity values in the range larger than 500 units (38.4\% compared to $11.7 \%$; < 0.001 ), whereas there was no significant difference in the intermediate grey intensity range between 250 and 500 units (39.3\% compared to $43.2 \% ; p=0.6$ ).

B. Avidity indices. The percentages of sera with avidity indices between 0 and 0.3 ( $71.8 \%$ versus $82.9 \%$ for hospitalized versus nonhospitalized patients), and 0.3-06 ( $16.2 \%$ versus $17.1 \%$ for hospitalized versus nonhospitalized patients) were not significantly different. However, a certain percentage of hospitalized patients reached avidity indices $>0.6(12 \%)$, whereas nonhospitalized patients did not reach these high avidity indices $(p<0.001)$.

These data show that hospitalized patients reach a substantial increase in antibody concentration (particularly after the first 25 days after onset of disease), whereas the increase in avidity is less substantial, though detectable with significance. 


\section{Figures}

Figure 1
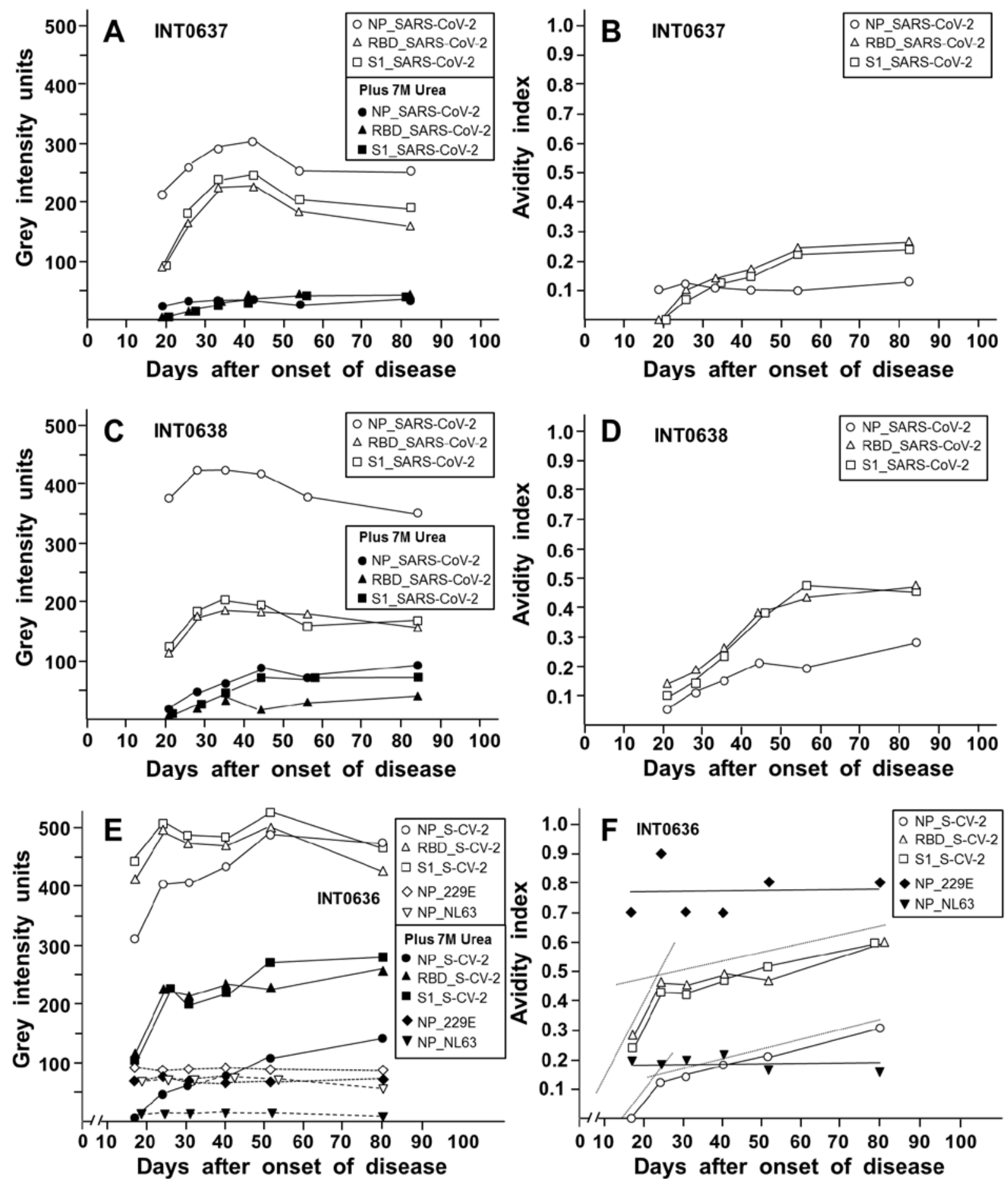
Figure 2
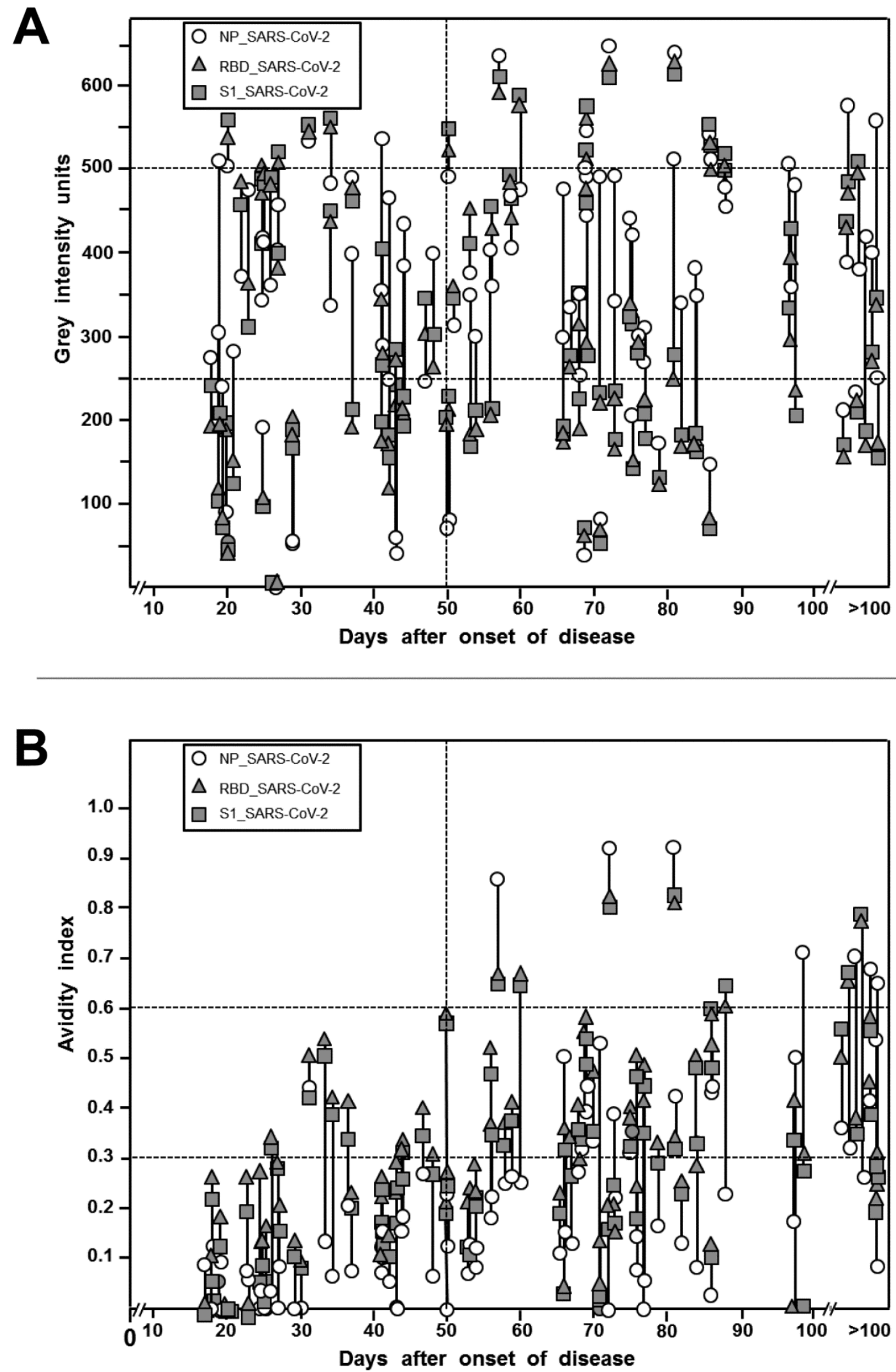
Figure 3
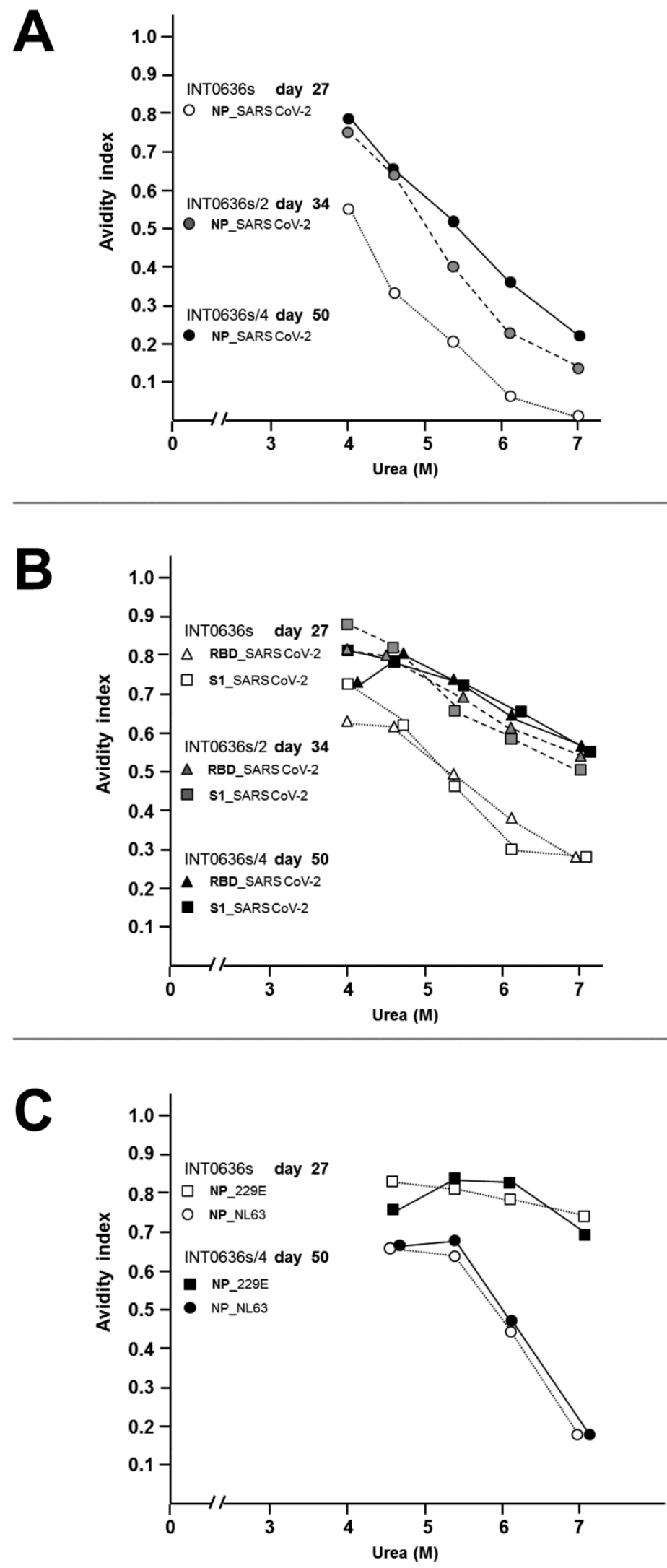
Figure 4
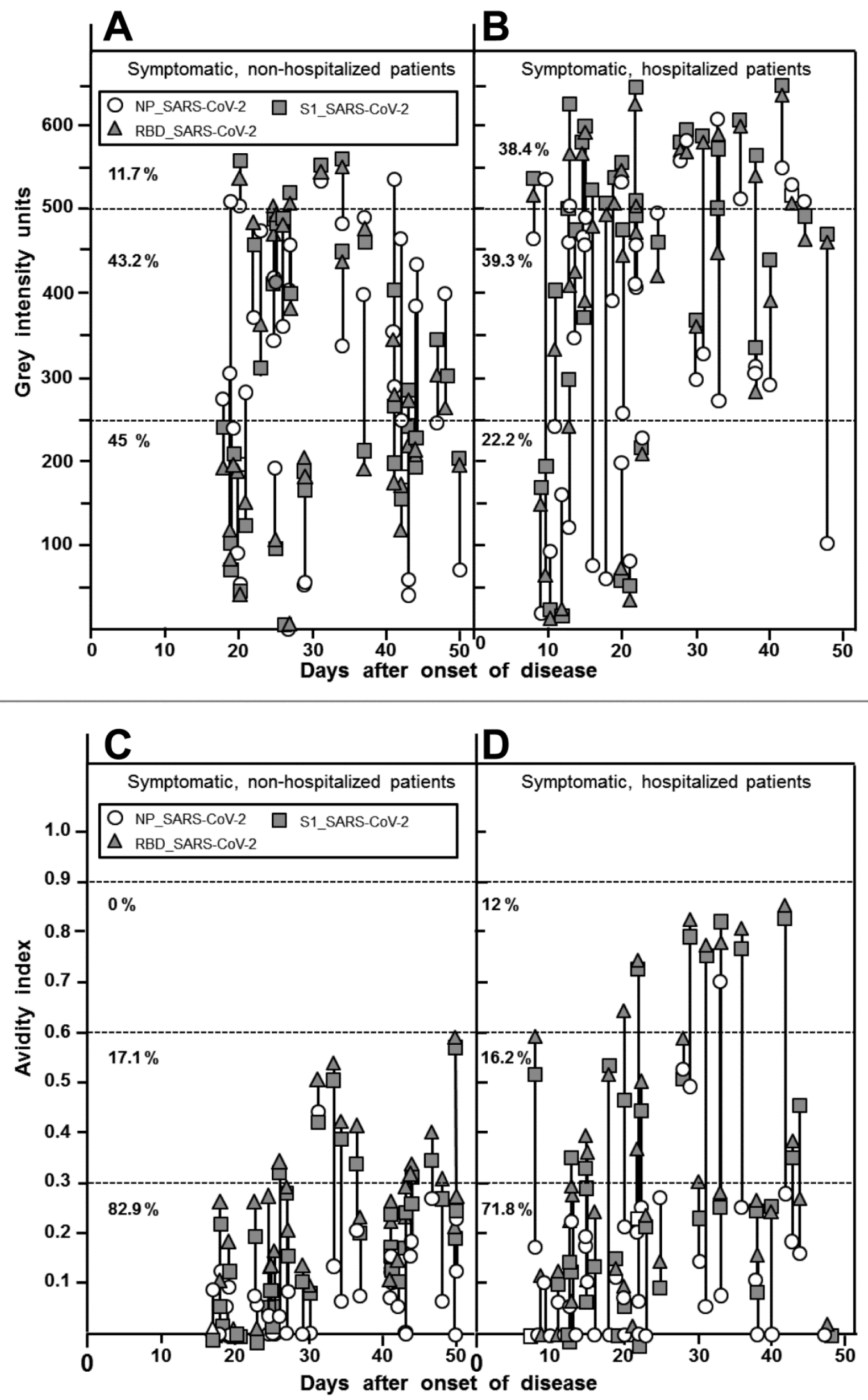


\section{Supplementary Materials}

The serological response to SARS corona virus-2 is characterized by frequent incomplete maturation of functional affinity (avidity)

Georg Bauer, Friedhelm Struck, Patrick Schreiner, Eva Staschik, Erwin Soutschek, Manfred Motz ${ }^{3}$

\section{Supplementary Materials summarize additional information on the following} aspects:

1. The role of avidity determination for virus serology

2. EBV serology as a prime example for avidity determination

3. Abrogation of avidity maturation after infection with seasonal corona viruses

4. Avidity maturation after SARS CoV-1 infections

5. Possible biological consequences from incomplete avidity maturation

6. Specific consequences for SARS CoV-2

7. Supplementary references

\section{The role of avidity determination for virus serology}

Within the germinal centers, proliferation of the IgG-producing B cells, hypermutation and clonal selection of $B$ cells that generate $\lg G$ with higher affinity, leads to the gradual increase in affinity of IgG generated by plasma cells derived from this cell population. Finally, memory B cells with the potential to rapidly generate specific IgG with high affinity mark the end point of successful affinity maturation. They assure a rapid humoral response to secondary infection and thus are of central importance for long-lasting protection (1-4). 
Affinity of IgG towards an antigen is defined by a process that summarizes the effects of binding of the antibody to the antigen and the release reaction of antibody from its target. This complete process cannot be easily measured in routine applications. Therefore, modern serology focused the analysis on determining the strength of the binding between antigen and IgG. The strength of interaction has been termed "avidity" (derived from latin aviditas, meaning desire, greed) or "functional affinity". It correlates well with total affinity. Therefore, the term "functional affinity" is also used for avidity. In routine diagnostics, avidity is measured by first allowing antibody-antigen interaction, and then testing the degree of release of antibody through the application of defined concentrations of chaotropic agents like urea. The determination of avidity starts with the incubation of serum with the test system in duplicate. After a washing step, one of the assays is immediately processed to quantification of bound IgG, whereas the parallel assay is first treated with defined concentrations of urea. After a subsequent washing step, this test is processed for quantification of bound IgG. An avidity index can be determined and used for differentiation between acute, intermediate and past infections by comparing the results between the urea-treated and the control assay. A strictly quantitative assay for the determination of the concentration of bound antibodies, as well as precision of the conditions of urea treatment are required for the determination of avidity. Furthermore, it is required that the concentration of antigen in the test system is not limiting, as otherwise antibodies of low avidity (affinity) are under-represented in the final outcome of the test, due to the dominant role of affinity for the binding process Immunoassays, particularly line assays, have been proven to very suitable for avidity determination for many reasons. First of all, this assay system allows to present different highly purified antigens on one test stripe and therefore to determine the 
avidities towards several defined antigens in the same test. (This advantageous test principle is protected by the international patent WO 00/54055; PCT/EP00/01883 and by EP 2019/2550 (pending)). The homogenous spreading of highly purified antigen in this test system is the basis for a homogenous signal, as the concentration of antibodies applied than faces a constant local concentration of antigens. This approach is different from assays based on classical western blots, where the antigen is distributed in the mode of a Gaussian distribution (as a result of movement of the antigens in a gel, immediately before application to the test system). This leads to variable conditions for the antibody/antigen interaction which is determined by the concentrations of the partners. The availability of sufficient quantities of highly purified recombinant antigen for preparation of the line assay allows to present a sufficiently large concentration of antigen. This allows the binding of antibodies with low and high affinity (avidity) in parallel and therefore allows to determine the degree of avidity maturation very precisely. If the antigen is supplied in limited concentrations only, as it occurs frequently with the use of immunofluorescence techniques, pure populations of antibodies with either very low or very high avidity can be easily discriminated, but low avidity antibodies are underrepresented during the course of avidity maturation.

Unfortunately, there is a certain degree of confusion in the literature, as the term "avidity" has been previously used to describe the complexity of an antibody population that is responsible for agglutination of a corpuscular target like bacteria. If the population contained antibodies directed towards different epitopes of the target structure, the association was more rigid. This historic type of "avidity" is mechanistically different from avidity as used in modern serology, especially as the 
complexity of the population does not have an impact on the binding strength of individual lgG molecules.

The second type of multiple use of the term "avidity" is related to the higher binding strength of bivalent antibody/antigen interaction (5) which may depend on the steric situation, compared to a monovalent binding. Again, this aspect is neither mechanistically nor conceptually related to the term "avidity" as used in the context of this manuscript.

It has to be kept in mind that antibody populations may be directed towards different epitopes and may have matured differentially with respect to avidity. Therefore, each antibody population has a diversity of binding specificities and strengths. These are independent of each other, but sum up in the measurement. This knowledge about diversity of the antibody population has to be kept in mind when the significance of kinetic measurements is being evaluated. In an ongoing process of avidity maturation, IgG of higher avidity will gradually replace the original low avidity lgG. However, for a certain period of time, essentially determined by the half-life of IgG, the previously generated low avidity lgG will still influence the overall outcome of avidity determination. Therefore, if avidity maturation was increasing, but then has stopped (as found for the IgG response towards SARS CoV-2 in this manuscript), the kinetics of avidity maturation will not go into a plateau immediately, as the disappearing $\lg G$ of lower affinity will cause the $\lg G$ of higher avidity to become more prominent, even though the maturation process has been already completed, but synthesis of high avidity antibodies is continued. An example of this phenomenon can be seen in Figure $1 \mathrm{~B}$ (for IgG towards $S 1$ and RBD) and in Figure 1D and F for all IgGs determined. If the stop in avidity maturation had occurred much earlier (Figure $1 \mathrm{~B}$, IgG towards NP of SARS CoV-2) or in Figure $1 \mathrm{~F}$ for IgG towards NP of 
the seasonal corona virus NL 63, where the infection with the seasonal corona virus was presumably before infection with SARS CoV-2, the kinetics of avidity is not increasing at all, but stays constantly at a low level.

There seems to be a consensus that high avidity is a primary requirement for efficient protection towards virus through humoral immunity (6-11). A rather good correlation between the neutralizing potential of IgG towards SARS CoV-2 S1 protein and its avidity has also been reported $(12,13)$. However, as shown by our data, an optimal degree of avidity maturation has not been achieved, despite a certain potential for neutralization.

\section{EBV serology as a prime example for avidity determination}

EBV serology was one of the first examples for the demonstration of a high degree of variability of the serological response (14). Due to the overlap of the symptoms of active EBV infection with many other disorders, clear serological differentiation was required. One step to achieve this goal was the differentiation between early markers, like IgG towards the viral capsid antigen (VCA) component p23 and late markers, like IgG towards EBNA-1 (p72) or a modified p18. Nevertheless, the serology of a certain percentage of cases remained unresolvable. The introduction of avidity determination into EBV serology has allowed to achieve a high degree of certainty in individual cases (14-17) and since then has been applied in the diagnosis in thousands of complex cases of potential EBV infections in the past. Therefore, avidity determination in EBV serology is a prime example to illustrate the potential, as well as limits of avidity determination in virus serology. The principle findings from this system can be directly applied to other viral systems. Its knowledge will be hopefully 
also useful for further refinement of SARS CoV-2 serology, despite its inherent problems due to frequent abrogation of avidity maturation.

Supplementary Figure 1 shows that the avidity of IgG towards p23 of EBV (determined with $7 \mathrm{M}$ urea in a line assay with recombinant antigens) is regularly low $(<0.6)$ within the first month after onset of disease, but reaches high values after two months. Therefore, a clear-cut differentiation between acute and past EBV infection is possible on the basis of avidity determination.

Supplementary Figure 1

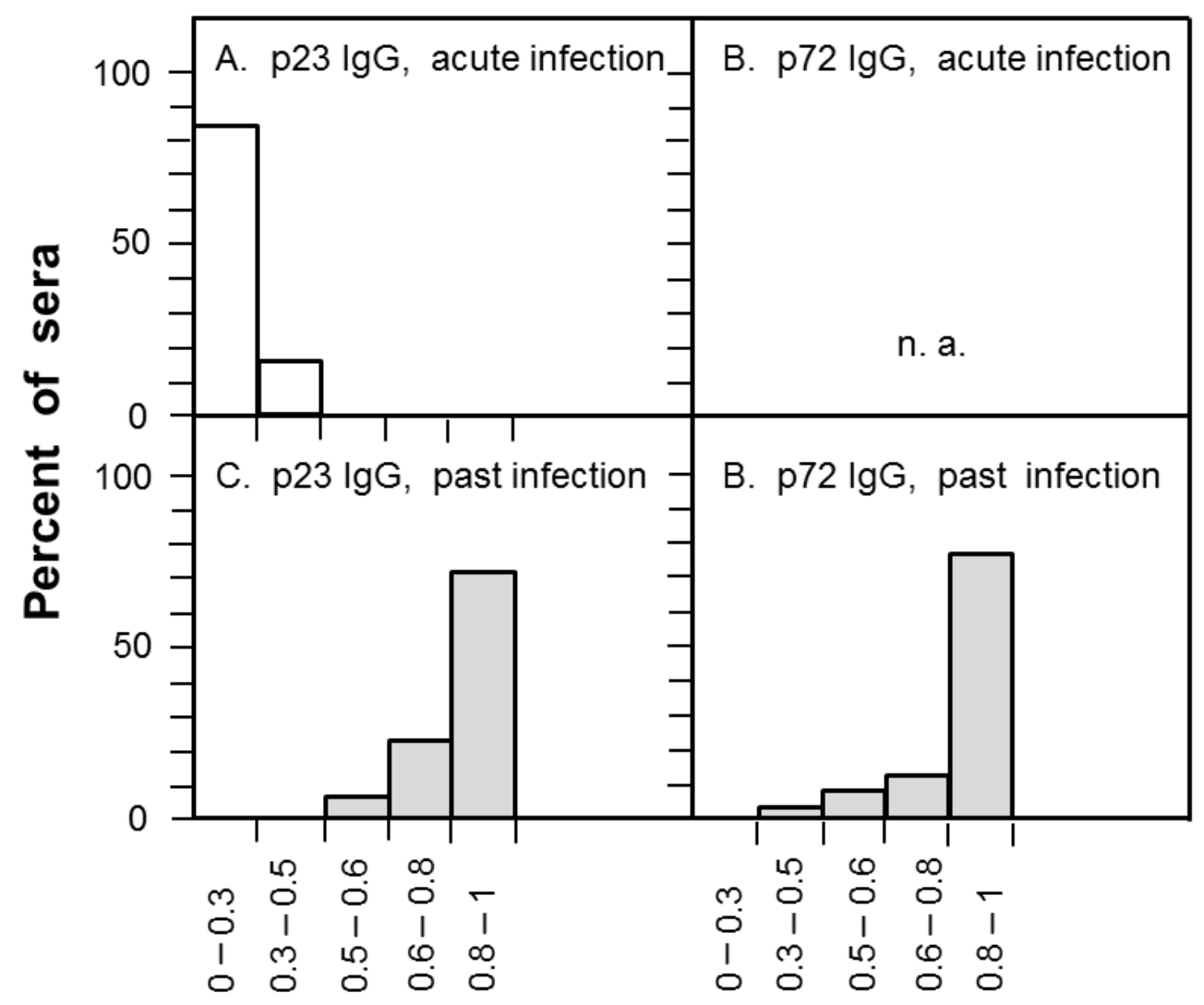

Avidity Index

Supplementary Figure 1: Avidity indices for IgG directed towards specific EBV antigens during acute and past EBV infection.

73 sera from acute EBV infection (taken less than four weeks after onset of symptoms and further defined by negative p72-lgG and p18-lgG) and 66 sera from 
past infection (taken more than two months past onset of symptoms and defined by positive p72-lgG) were tested in the recomLineEBV avidity assay (Mikrogen, article nr. 4572) following the protocol supplied by Microgen and described in references \# 14-17. The data show that acute EBV infections are uniformly characterized by low avidity of p23 IgG, whereas past EBV infections reach avidity indices higher than 0.5 . Furthermore, p72 lgG is missing early in acute infection and is of high avidity in past infection. This pattern allows clear cut serodiagnosis for the differentiation of acute and past EBV infection.

The usefulness of this application is further illustrated in Supplementary Figures 2-4. Supplementary Figure $2 \mathrm{~A}$ shows that acute EBV infections lack both late markers p72-lgG and p18 IgG (14). Urea treatment for avidity determination (Supplementary Figure 2 B) shows the low avidity of p23 IgG in these cases, as the urea treatment causes substantial removal of bound IgG. Supplementary Figure 2B also shows that avidity determination of the early antigens p138 and p54 is not useful for avidity determination, as these markers are developed very early and therefore may have reached high avidity already at the onset of disease.

Classical past EBV infections are characterized by expression of both late markers p72 IgG and p18 IgG, which, like p23 IgG, all show high avidity (Supplementary Figures $3 \mathrm{~A}, \mathrm{~B})$. The late stage of infection is confirmed in these samples by the high avidities of IgG directed towards p23, p18 and p72. Interestingly, the avidity of IgG towards the early antigens p138 and p54 is low in a substantial number of these cases of past EBV infection. This finding indicates immature avidity maturation of IgG towards p138 and p54, which is in line with the very low concentrations of these early antigens reached during infection or reactivation, as p138 and p54 represent regulatory proteins which are only required in low copy numbers. 
One of the central problems in EBV serology is the lack of development of anti-p72 in rare cases, which represented about $5 \%$ of patients tested. The lack of p72-lgG was often mistaken as an indication of ongoing acute EBV infection. This diagnostic problems has been resolved through the application of avidity determination (Supplementary Figure 4 A, B). Here, high avidity of IgG towards p23 (despite lacking p72-lgG) points to past infection and allows for a clear cut differentiation from acute infections, where p23 IgG is of low avidity, as shown before in Supplementary Figures $2 \mathrm{~A}$ and $\mathrm{B}$.

The comparison of the data established for EBV with those determined for SARS CoV-2 in this manuscript illustrates the incomplete avidity maturation in the majority of cases of SARS CoV-2 infections, which is contrasted by the regularly occurring complete maturation in the case of EBV infection. The picture shown for EBV represents the general picture, which is also found in other viral infections $(3,18-25$; summarized in ref. \# 26), whereas the pattern of avidity maturation after SARS CoV-2 seems to be the exception.

Our data exclude that low avidity found for SARS CoV-2 IgG is simply due to a technical problem or unsuitability of SARS CoV-2 antigen for the test procedure, as high avidity was indeed reached and was measurable in a certain percentage of samples.

Rather, as an alternative and rational explanation, incomplete maturation of IgG towards SARS CoV-2 antigens might be due to a restricted availability of SARS CoV2 antigen for the immune system of infected patients. Incomplete avidity maturation of IgG towards SARS CoV-2 has a strong impact on SARS CoV-2 serodiagnosis, on the understanding of susceptibility towards reinfection and lack of herd immunity, as well as for specific challenges related to immunization programs. 
Supplementary Figure 2 A

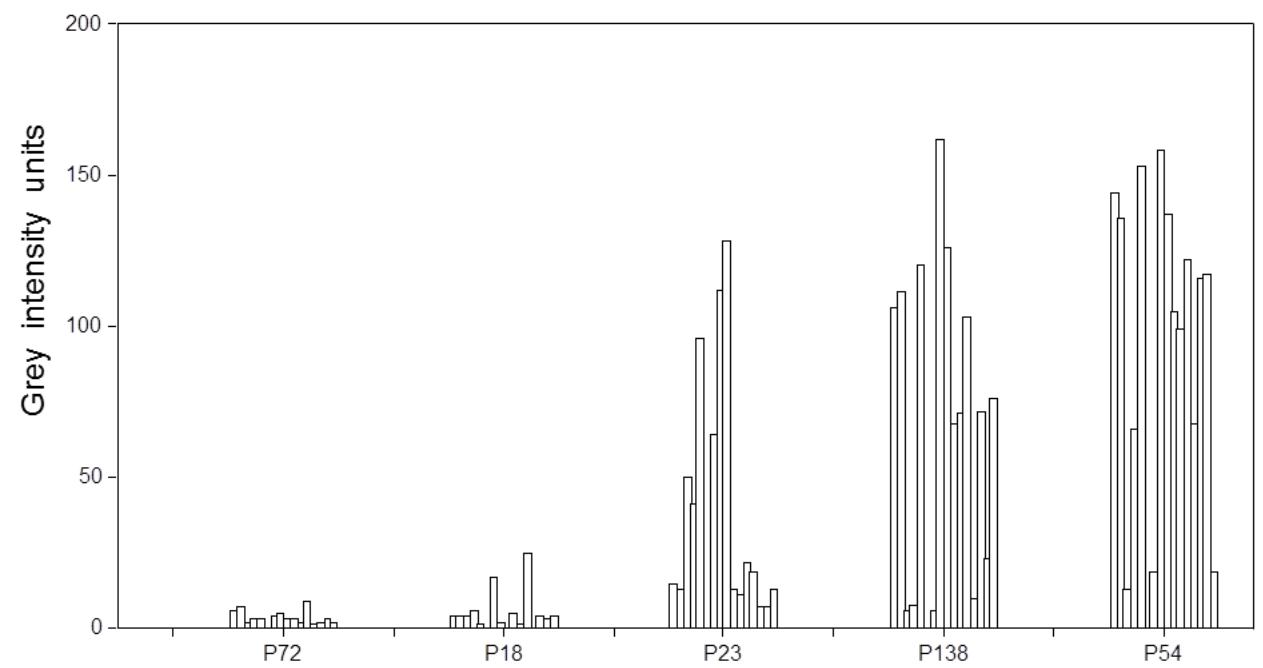

Supplementary Figure 2 B.

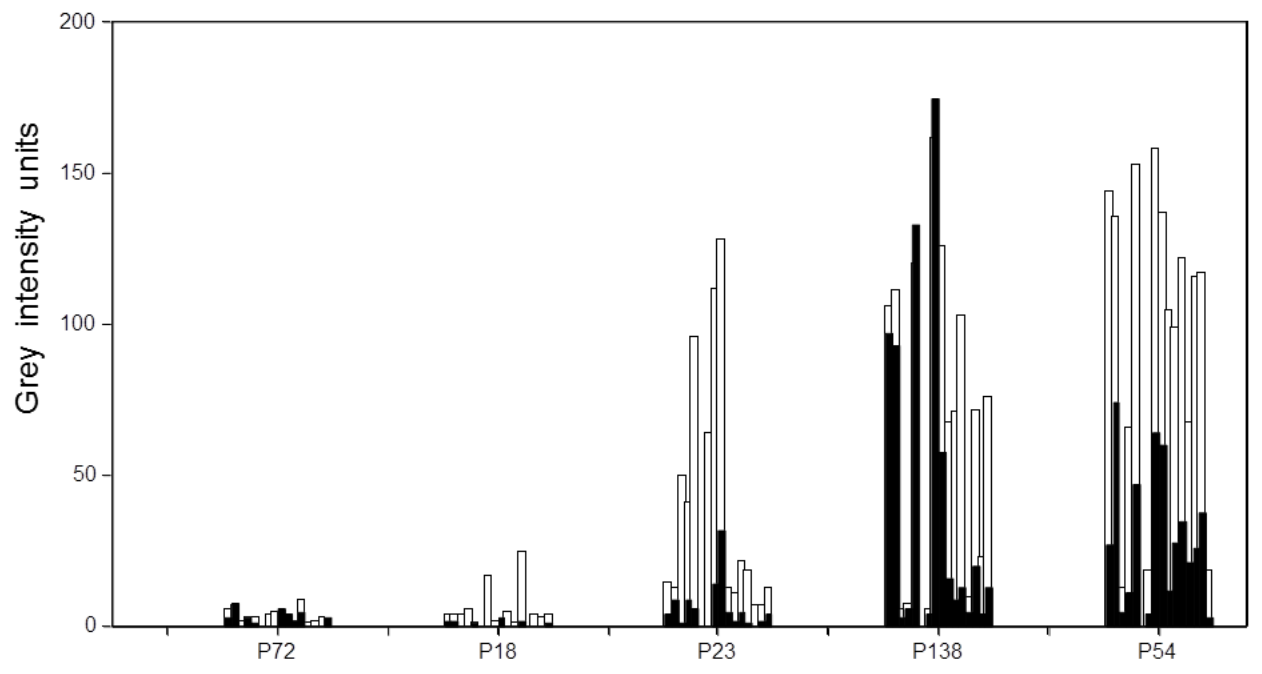

Supplementary Figure 2 A and B: Acute EBV infections are characterized by the lack of $p 72-\lg G$ and $p 18-\lg G$ and the presence of p23-lgG, p138-lgG and p4- $\lg G$. Urea treatment after the incubation of serum with the immunoblot stripes leads to removal of bound low avidity IgG. The remaining IgG is indicated by black label under B. 
Supplementary Figure $3 \mathrm{~A}$

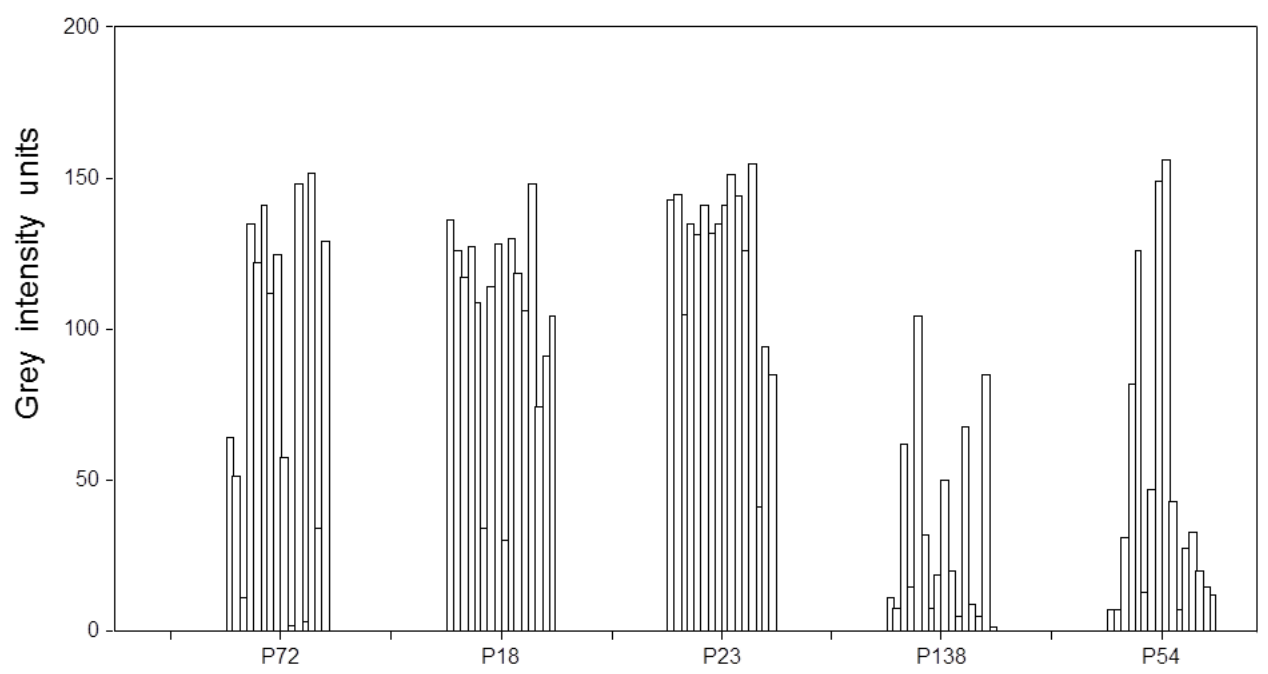

Supplementary Figure 3 B

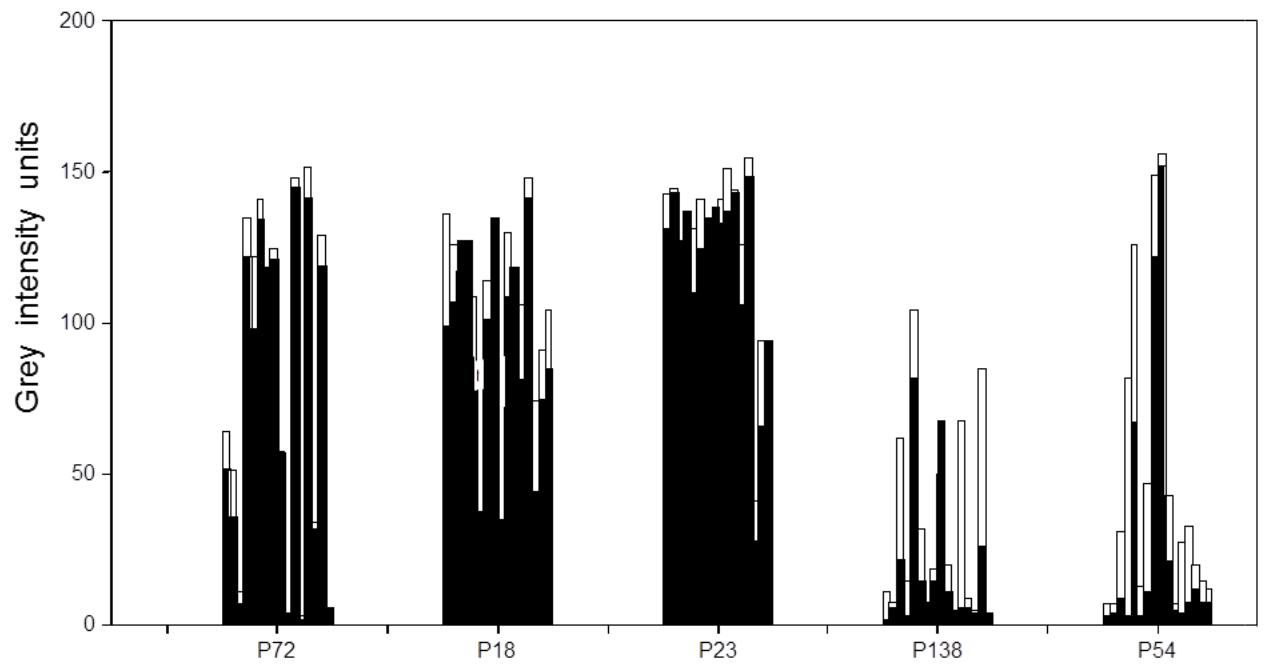

Supplementary Figure 3 A and B: Past EBV infections are characterized by positive p72$\lg G, p 18-\lg G$ and $p 23-\lg G$ of high avidity. IgG remaining after urea treatment is indicated by black label under B. Please note the presence of p138-lgG and p54-lgG of immature avidity. 
Supplementary Figure $4 \mathrm{~A}$

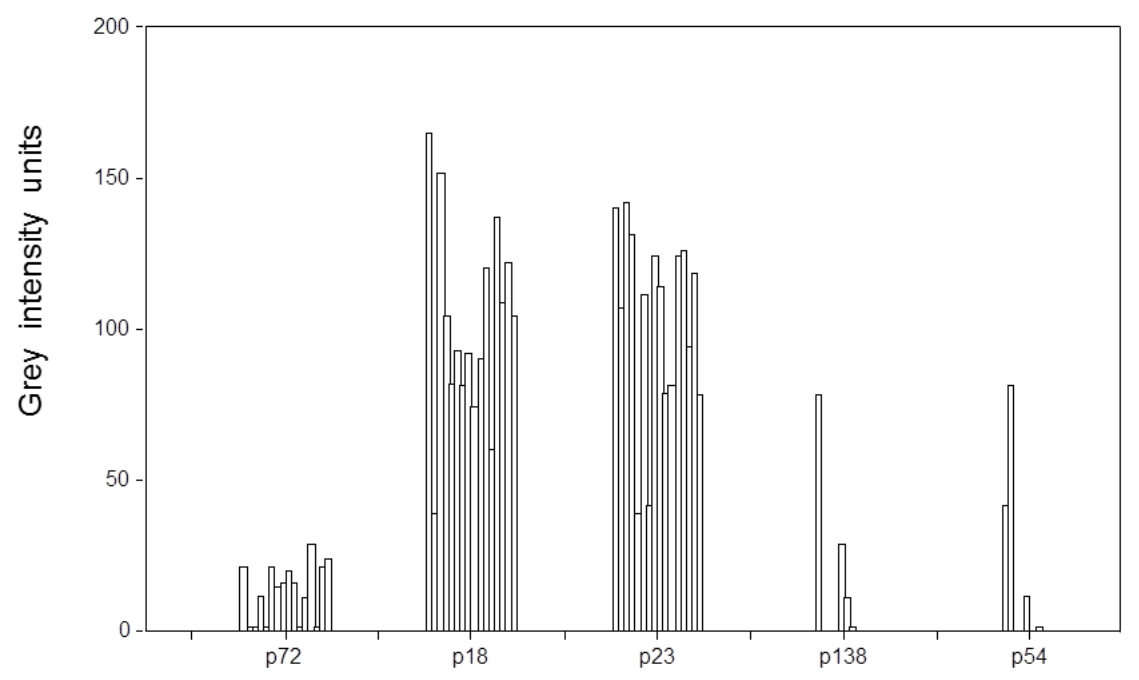

Supplementary Figure 4 B

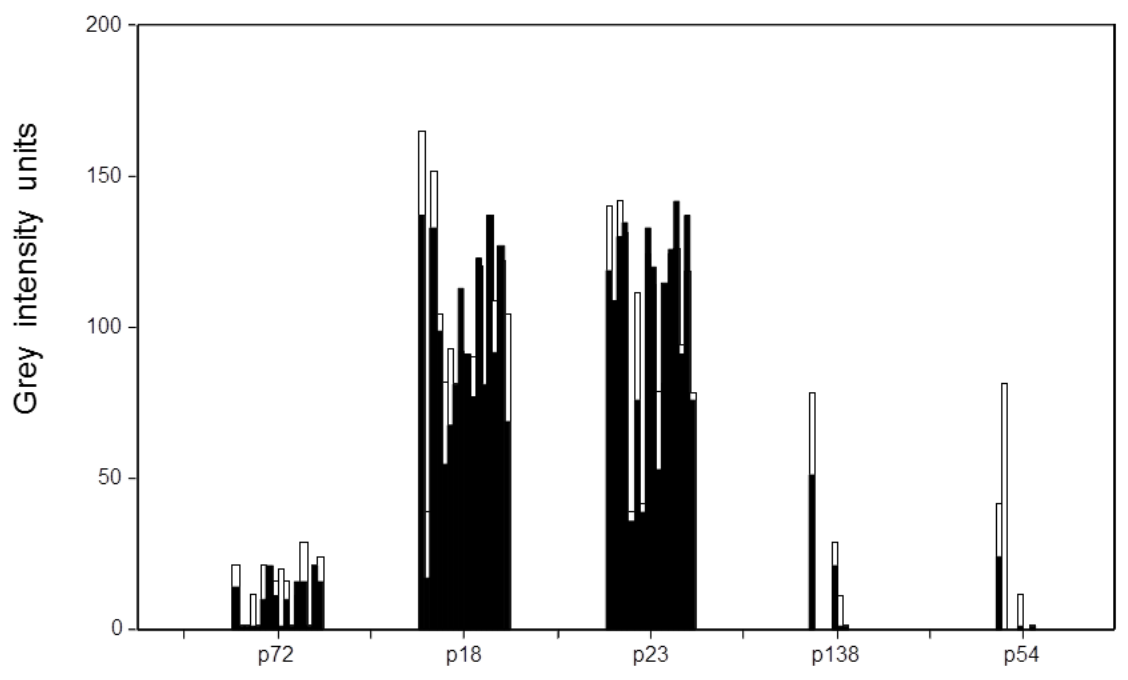

Supplementary Figure 4 A and B: In rare cases of past infections (about $5 \%$ of all infections), the late marker p72-lgG is not developed. However, the high avidity of $p 18-\lg G$ and p23-IgG allows to recognize the correct status "past infection".. 


\section{Abrogation of avidity maturation after infection with seasonal corona viruses}

The test system used in this study not only used SARS CoV-2 NP, S1 and RBD, but also NP of the four seasonal corona viruses $229 \mathrm{E}, \mathrm{NL63}, \mathrm{OC} 43$ and HKU1. The parallel determination of IgG towards SARS CoV-2 as well as seasonal corona viruses gives the chance to determine the true serostatus in case of potential crossreactions or cross stimulations of the immune responses.

Supplementary Figure 5 shows the grey intensity units for IgG directed towards the NPs of these four seasonal corona viruses that had been determined in parallel to the SARS CoV-2-specific IgG in the cases presented in Figure 2 of the main manuscript. Not all of the patients had IgG towards seasonal corona virus NP, and those that were positive did not show a recognizable pattern of combination. Supplementary Figure 5 shows that the grey intensity values that were reached and represented the concentration of IgG were below 200 grey intensity units in most cases, whereas most of the OD values for Anti-SARS CoV-2 IgG in Figure 2 A were higher than 200 grey intensity units $(p<0.001)$. There was no significant difference between the grey intensity values reached for the four different seasonal corona viruses.

The situation was completely different, when the avidity indices of the IgG towards NP of seasonal corona viruses were determined (Supplementary Figure 6). In the case of $229 \mathrm{E}$ and NL63, more than two thirds of the sera showed high avidity (avidity index $>0.6)$ and less than one third low avidity $(p<0.001)$. There was no significant difference with respect to this pattern for $229 \mathrm{E}$ and NL63. However, the results for this group of viruses was completely different from the group consisting of OC43 and HKU1 $(p<0.001)$, where the ratio between high and low avidity was reversed compared to the other group. These data show i) that incomplete maturation of avidity is also found after infection with all four seasonal corona viruses, and ii) that 
the degree of incomplete maturation is strongly different between different members of this family of viruses. One possible explanation for the more pronounced avidity maturation in the case of $229 \mathrm{E}$ and NL63 might be a more frequent repeated contact with these viruses, leading to gradual increase in avidity with time. Alternatively, for unknown reasons so far, the infections with 229E and NL63 might lead to higher and more prolonged accessibility of viral antigens for the immune system compared to SARS CoV-2 infections. These assumptions are merely theoretical, so far. The resolution of these aspects requires further analysis.

\section{Supplementary Figure 5}

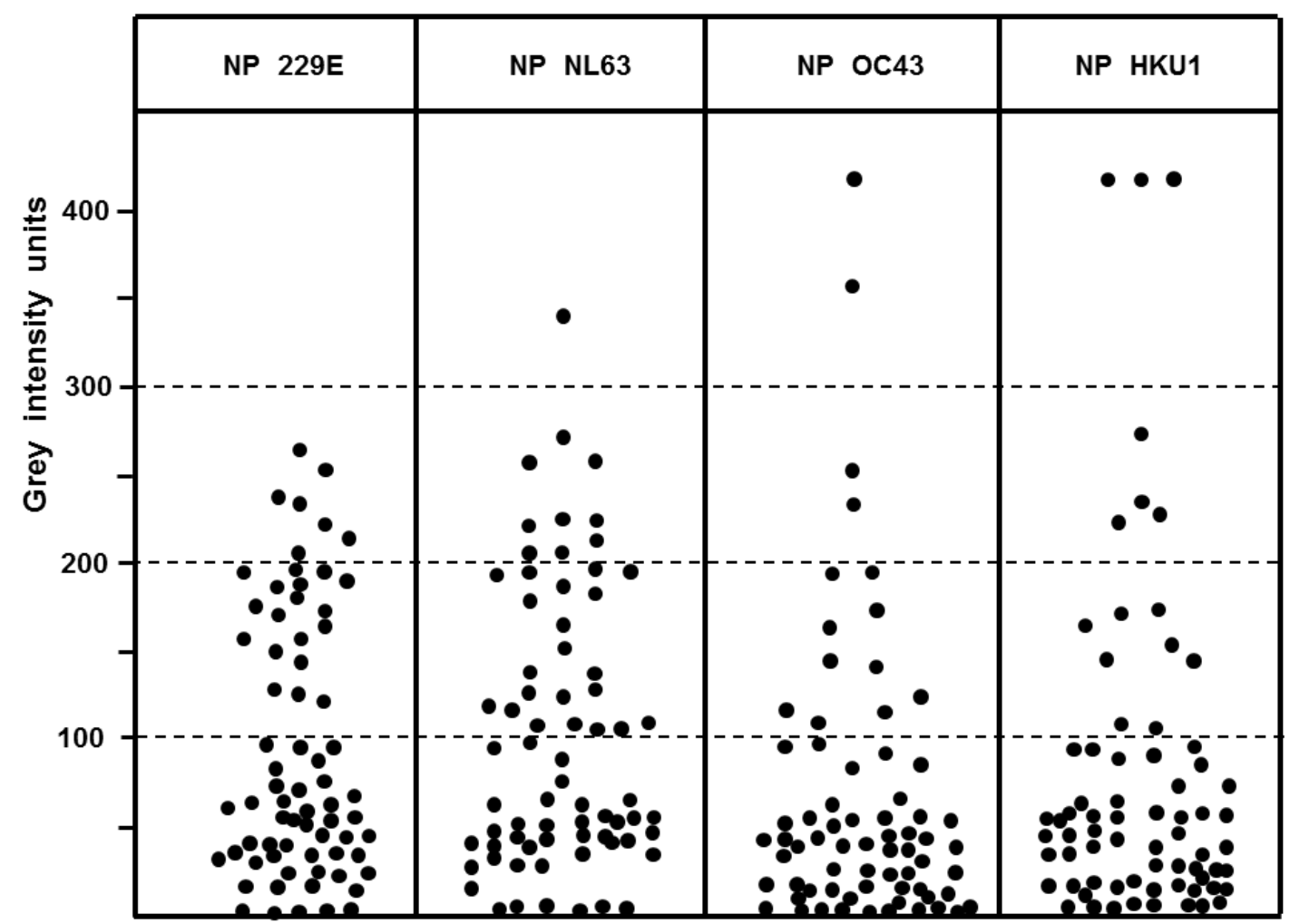

Supplementary Figure 5. Grey intensity values of IgG directed towards the NPs of the four seasonal corona viruses 229E, NL63, OC43 and HKU1. The values were determined in parallel to IgGs towards SARS CoV-2 in 93 sera of COVID-19 patients, as described in Figure 2 of the main manuscript. 
Supplementary Figure 6

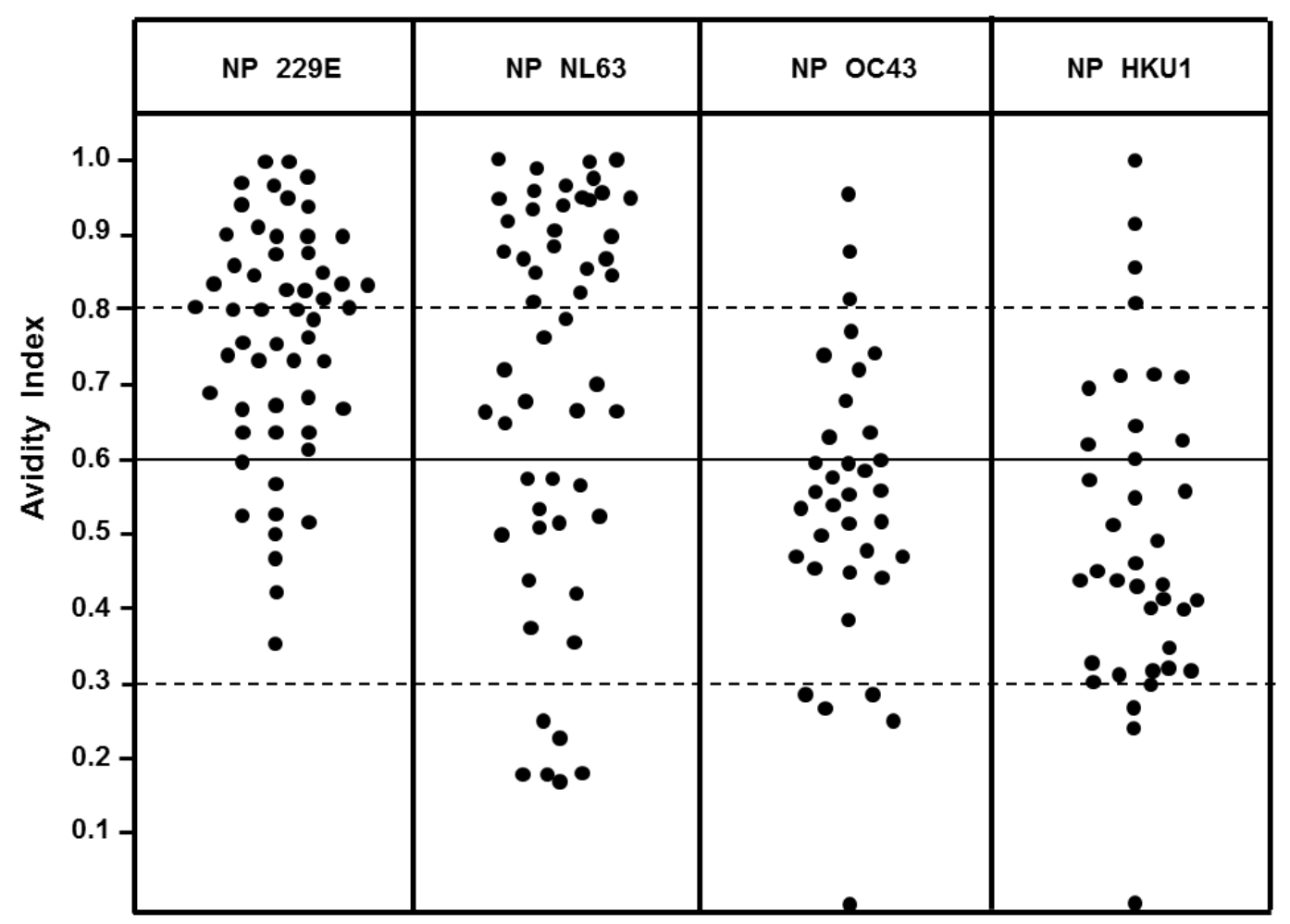

Supplementary Figure 6. Avidity indices of IgG directed towards the NPs of the four seasonal corona viruses 229E, NL63, OC43 and HKU1. The values were determined in parallel to IgGs towards SARS CoV-2 in 93 sera of COVID-19 patients, as described in Figure 2 of the main manuscript.

\section{Avidity maturation after SARS CoV-1 infections}

Avidity determination has been previously applied to the serodiagnosis of SARS

CoV-1 infections during the pandemics in 2002/2003 (27). Chan et al. reported that all patients reached high avidity after approximately 80 days past the onset of fever. At first sight, this finding seems to indicate that the immune response towards SARS CoV-1 reached complete maturation and thus was different from the response found towards SARS CoV-2 during the recent pandemics, as reported here and elsewhere $(12,28,29)$. However, the avidity determination by Chan et al. (27) was based on the application of $4 \mathrm{M}$ urea rather than $7 \mathrm{M}$ urea, as used in our study. At this relatively 
low concentration of $4 \mathrm{M}$ urea, IgG with intermediate avidity is no longer removed from the antigen, thus mimicking high avidity. However, the data by Chan et al.(27) demonstrate that avidity of IgG directed towards SARS CoV-2 matures to higher avidities over long times.

\section{Possible biological consequences from incomplete avidity maturation}

Based on the findings that high avidity $\lg G$ is required for efficient protection towards infection after immunization or reinfection, incomplete avidity maturation of the immune response towards corona viruses, particularly their S1 protein, including the receptor binding domain $(\mathrm{RBD})$ might indicate that the establishment of an $\lg \mathrm{G}$ response is not sufficient in all cases to prevent reinfection. Interestingly, our finding on low avidity of IgG towards seasonal corona viruses correlates very well with the frequent reinfection with seasonal corona viruses $(30,31)$. This phenomenon has been particulary determined for OC43 and HKU1. Though only the response towards NP of seasonal corona viruses has been determined, it may be assumed that the immune response towards S1 and RBD follows a similar pattern - similarly to the findings for the immune response towards SARS CoV-2. These findings allow to speculate that the strategy of corona viruses in general is to establish a rather low protective immune response, thus allowing repeated cycles of infection from year to year. The data also show, that the IgG of low avidity does have a limiting effect on virus replication during initial infection, potentially due to a short term higher concentration. However, the lack of avidity maturation might ensure that the immune system is not fit to defend a secondary attack by the same virus later, when the initial concentration of IgG has been fallen, and no memory cells with the potential to generate high avidity antibodies are existing. Based on the mechanism of avidity maturation, this scenario can be controlled by the virus by a low degree of replication 
and spread into the body. Its local replication in the respiratory system ensures its transmission, but also seems to be suboptimal for providing sufficient antigen to ensure a complete process of avidity maturation. It is noted that additional effects of the cellular immune system can be expected. So far we have no data on these cellular aspects.

\section{Specific consequences for SARS CoV-2}

As SARS CoV-2 shows a similar strategy with respect to prevention of avidity maturation as its seasonal viral relatives, it might have the potential to become a new seasonal corona virus. This scenario is frightening for several reasons. Therefore, immunization programs need to make sure that the immune response after immunization is not only strong, i. e. high in IgG titer, but also in quality, i. e. it should reach high avidity.

As patients with more severe COVID-19 showed a higher probability of high avidity IgG towards SARS CoV-2 than patients with milder symptoms, we may be optimistic that high avidity can be reached. The clue for this goal most likely is connected to the right and sustained concentration of viral antigen during immunization and the right schedule of immunization. The determination of avidity, based on the method described in this paper, might help to control this process in a satisfactory mode.

\section{Supplementary references}

1 Eisen HN, Siskind GW. Variations in the affinities of antibodies during the immune response. Biochemistry 1964; 3: 996-1008.

2 Foote J, Milstein C. Kinetic maturation of an immune response. Nature 1991; 352: 530-531.

3 Hedman K, Lappalainen M, Söderlund M, Hedman L. Avidity of IgG in serodiagnosis of infectious diseases. Rev Med Microbiol 1997; 4: 123-129. 
4 Hazel SL. Clinical utility of avidity assays. Expert Opinion on medical diagnostics 1: 511519,2007

5 Liu H, Wu NC, Yuan M, Bangaru S, Torres JL, Caniels TG, van Schooten J, Zhu X, - Lee C-CL, Brouwer PJM, van Gils MJ, Sanders RW, Ward AB, Wilson IA. Crossneutralization of a SARS-CoV-2 antibody to a functionally conserved site is mediated by avidity. bioRxiv preprint doi: https://doi.org/10.1101/2020.08.02.233536

6 Junker AK, Tilley P. Varicella-zoster virus antibody avidity and subclass patterns in children with recurrent chickenpox. J Med Virol 1994; 43: 119-124.

7 Martin KA, Junker AK, Thomas EE, Van Allen MI, Friedman, JM. Occcurence of chickenpox during pregnancy in women seropositive for varicella-zoster virus. J Infect Dis 1994; 170: 991-995.

8 Boppana SB, Britt WJ. Antiviral antibody responses and intrauterine transmission after primary maternal cytomegalovirus infection. J Infect Dis 1995; 171: 1115-1121.

9 Delgado MF, Coviello S, Monsalvo AC, Melendi GA, Hernandez JZ, Batalle JP, Diaz L, Trento A, Chang H-Y, Mitzner W, Ravetch J, Melero JA, Irusta PM, Polack FP. Lack of antibody affinity maturation due to poor Toll-like receptor stimulation leads to enhanced respiratory syncytial virus disease. Nature Medicine, 2009; 15: 34-41.

DOI 10.1099/jgv.0.001439

10 Kontio M, Jokinen S, Paunio M, Peltola H, Davidkin I. Waning antibody levels and avidity: implications for MMR vaccine-induced protection. JID 2012; 206: 1542-1548.

11 Puschnik A, Lau L., Cromwell EA., Balmaseda A, Zompi S, Harris E. Correlation between Dengue-specific neutralizing antibodies and serum avidity in primary and secondary Dengue virus 3 natural infections in humans.PLOS Neglected Tropical Diseases, 2013; 7: e2274

12 Benner S, Patel EU, Laeyendecker O, Pekosz A, Littlefield K, Eby Y, Fernandez RE, Miller J, Kirby CS, Keruly M, Klock E, Baker OR, Schmidt HA, Shrestha R, Burgess I, Bonny TS, Clarke W, Caturegli P, Sullivan D, Shoham S, Quinn TC, Bloch EM, Casadevall A, Tobian AAR, Redd AD. SARS-CoV-2 antibody avidity responses in covid-19 patients and convalescent plasma donors. The Journal of Infectious Diseases, jiaa581, https://doi.org/10.1093/infdis/jiaa581

13 Klein SL, Pekosz A, Park H-S, Ursin RL, Shapiro JR, Benner SE, Littlefield K, Kumar S, Naik HM, Betenbaugh MJ, Shrestha R, Wu AJ, Hughes RM, Burgess I, Caturegli P, Laeyendecker O, Quinn TC, Sullivan D, Shoham S, Redd AD, Bloch EM, Casadevall A, Tobian AAR.Sex, age, and hospitalization drive antibody responses in 1 a COVID-19 convalescent plasma donor population. J Clin Invest. 2020.

https://doi.org/10.1172/JCl142004

14 Niller HH, Bauer G. Epstein-Barr Virus: Clinical Diagnostics. Meth Mol. Biol 2017; 1532: 33-55. 
15 Bauer G. Simplicity through complexity: immunoblots with recombinant antigens as the new gold standard in Epstein Barr virus serology. Clin. Lab. 47: 223-230, 2001.

16 Pottgiesser T, Schumacher Y O, Wolfarth B, Schmidt-Trucksäss A and Bauer G. Longitudinal observation of Epstein-Barr virus antibodies in athletes and controls during a competition season. J. Med. Virol. 84: 1415-1422, 2012.

17 Pottgiesser T, Wolfarth B, Schumacher YO and Bauer G. Epstein-Barr virus serostatus: no difference despite aberrant patterns in athletes and control groups. Med \& Science in Sports and Exercise 38: 1782-1791, 2006.

18 Hedman K, Rousseau SA. Measurement of avidity of specific IgG for verification of recent primary rubella. J Med Virol 1989; 27: 288-292. doi.org/10.1002/jmv.1890270406

19 Hedman K, Seppalä I. Recent rubella infection indicated by a low avidity of specific IgG. J Clin Immunol 1988; 8: 214-221.

20 Robertson P, Beynon S, Whybin R, Brennan C, Vollmer-Conna U, Hickie I, Lloyd A. Measurement of EBV-IgG anti-VCA avidity aids the early and reliable diagnosis of primary EBV infection. J Med Virol 2003; 70:617-623.

21 Gassmann C, Bauer G. Avidity determination of IgG directed against tick-borne encephalitis virus improves detection of current infections. J Med Virol 1997; 51: 242-251.

22 Lazzarotto T, Varani S, Spezzacatena P, Gabriell L, Pradelli P, Guerra B, Landini MP. Maternal IgG Avidity and IgM Detected by Blot as Diagnostic Tools to Identify Pregnant Women at Risk of Transmitting Cytomegalovirus. Virol Immunol 13: 137-141, 2009. https://doi.org/10.1089/vim.2000.13.137

23 Ward KN, Turner DJ, Couto Parada X, Thiruchelvam AD. Use of immunoglobulin G antibody avidity for differentiation of primary human herpesvirus 6 and 7 infections. J Clin Microbiol 2001; 39: 959-963. DOI: 10.1128/JCM.39.3.959-963.2001

24 Söderlund M, Brown C, Cohen BJ, Hedman K. Accurate serodiagnosis of B 19 parvovirus infections by measurement of IgG avidity. JID 1995; 171: 710-713.

25 Paunio M, Hedman K, Davidkin I, Peltola H. IgG avidity to distinguish secondary from primary measles vaccination failures: prospects for a more effective global measles elimination strategy. Exp Opininion Pharmacotherapy 2005; 4: 1215-1225.

26 Bauer G. The variability of the serological response to SARS-corona virus-2: Potential resolution of ambiguity through determination of avidity (functional affinity). J Med Virol. 2020;1-12.https://doi.org/10.1002/jmv.26262

27 Chan PKS, Lim P-L, Liu EYM, Cheung JLK, Leung DTM, Sung JJY. Antibody avidity maturation during severe acute respiratory syndrome-associated coronavirus infection. JID, 2005; 192:166-9. 
28 Strömer A, Grobe O, Rose R, Fickenscher H, Lorentz T, Krumbholz A. Diagnostic accuracy of six commercial SARS-CoV-2 lgG/total antibody assays and identification of SARS-CoV-2 neutralizing antibodies in convalescent sera. medRxiv doi:

https://doi.org/10.1101/2020.06.15.20131672

29 Strömer A, Rose R, Grobe O, Neumann F, Fickenscher H, Lorentz T, Krumbholz A. Kinetics of nucleo- and spike protein-specific immunoglobulin $G$ and of virus-neutralizing antibodies after SARS-CoV-2 infection. Microorganisms 2020, 8, 1572;

doi: $10.3390 /$ microorganisms 8101572

30 Edridge AWD, Kaczorowska J, Hoste ACR, Bakker M, Klein M, Maarten F, Jebbink MF, Matser A, Kinsella CM, Rueda P, Prins M, Sastre P, Deijs M, van der Hoek L.

Human coronavirus reinfection dynamics: lessons for SARS-CoV-2. medRxiv preprint doi: https://doi.org/10.1101/2020.05.11.20086439

31 Galanti M and Shaman J. Direct observation of repeated infections with endemic coronaviruses. The Journal of Infectious Diseases, jiaa392, 2020;

https://doi.org/10.1093/infdis/iiaa392 

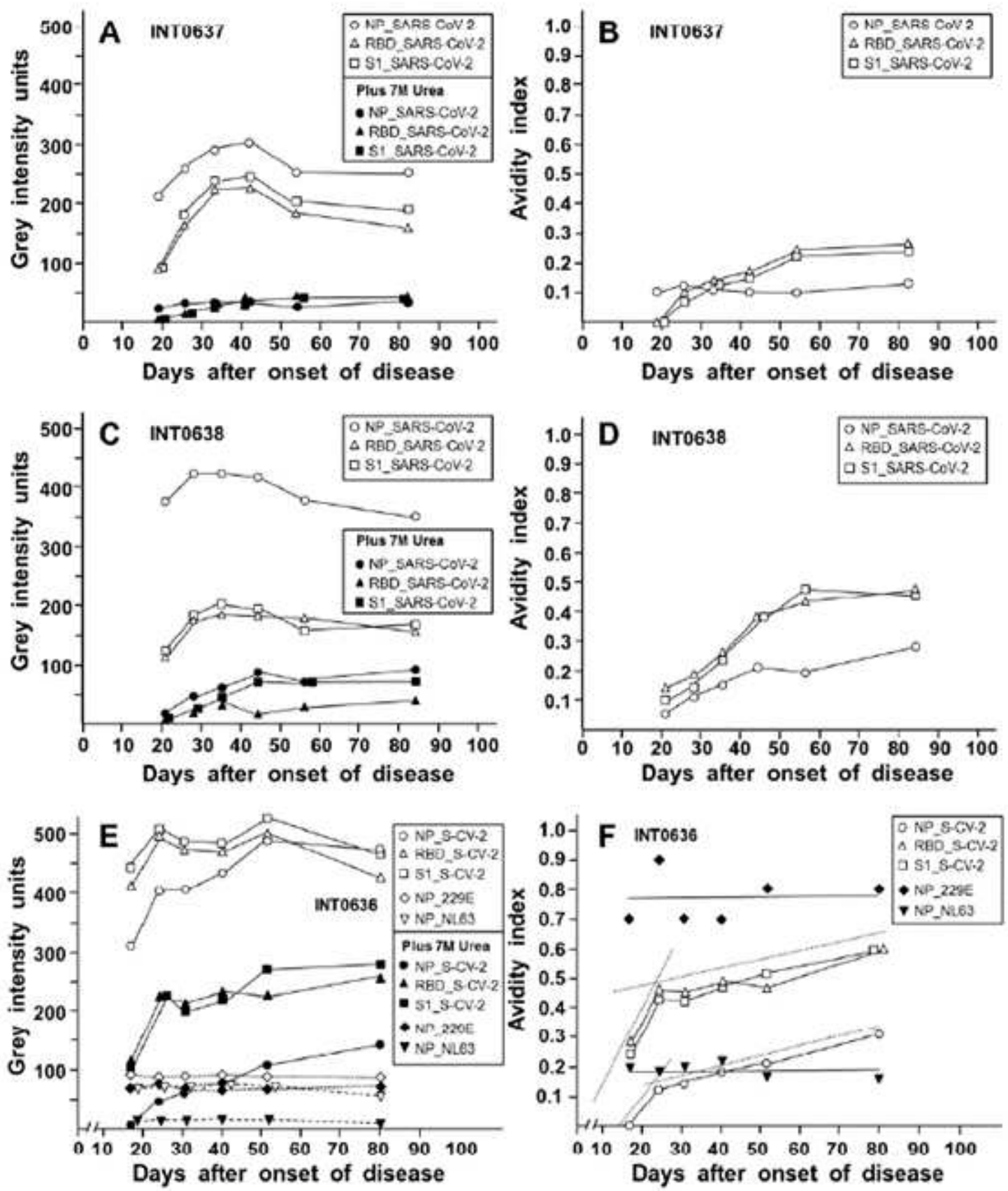

\section{Figure 1}

Kinetics of the serological responses to SARS CoV-2 nucleoprotein (NP), receptor-binding domain (RBD) and surface protein S1. Sera from three patients with COVID-19 confirmed by positive PCR and showing clinical symptoms were tested for IgG towards SARS CoV-2 NP, RBD and S1, as well as towards NP of the seasonal coronaviruses $229 \mathrm{E}, \mathrm{NL63}$; OC43 and HUK1 in the determination described under E and F, using a recombinant line immunoassay. The immunoassays were performed without and with urea treatment (7M) for the determination of avidity. A, C, and E shows the grey intensity values determined, whereas B, 
$D$, and $F$ show the calculated avidity indices. The data show an initial increase of grey intensities for IgG towards the SARS CoV-2 proteins, which is followed by a plateau and even an indication of decline. Avidity maturation of the IgGs towards SARS CoV-2 is variable, but overall poor. The avidity index for IgG towards NP in B constantly stays at 0.1 , whereas the other two IgGs show initial marginal avidity maturation, ending at a plateau of 0.2 . The examples under $D$ and $F$ are characterized by an initial increase of avidity, followed by a plateau or only slight increase. Avidity of IgG towards seasonal coronaviruses was either of high avidity (D), or of high avidity towards NP of 229E and constantly low avidity towards NL63. These findings demonstrate that incomplete avidity maturation is characteristic for the response towards SARS CoV-2 and can also be found for other corona viruses. 

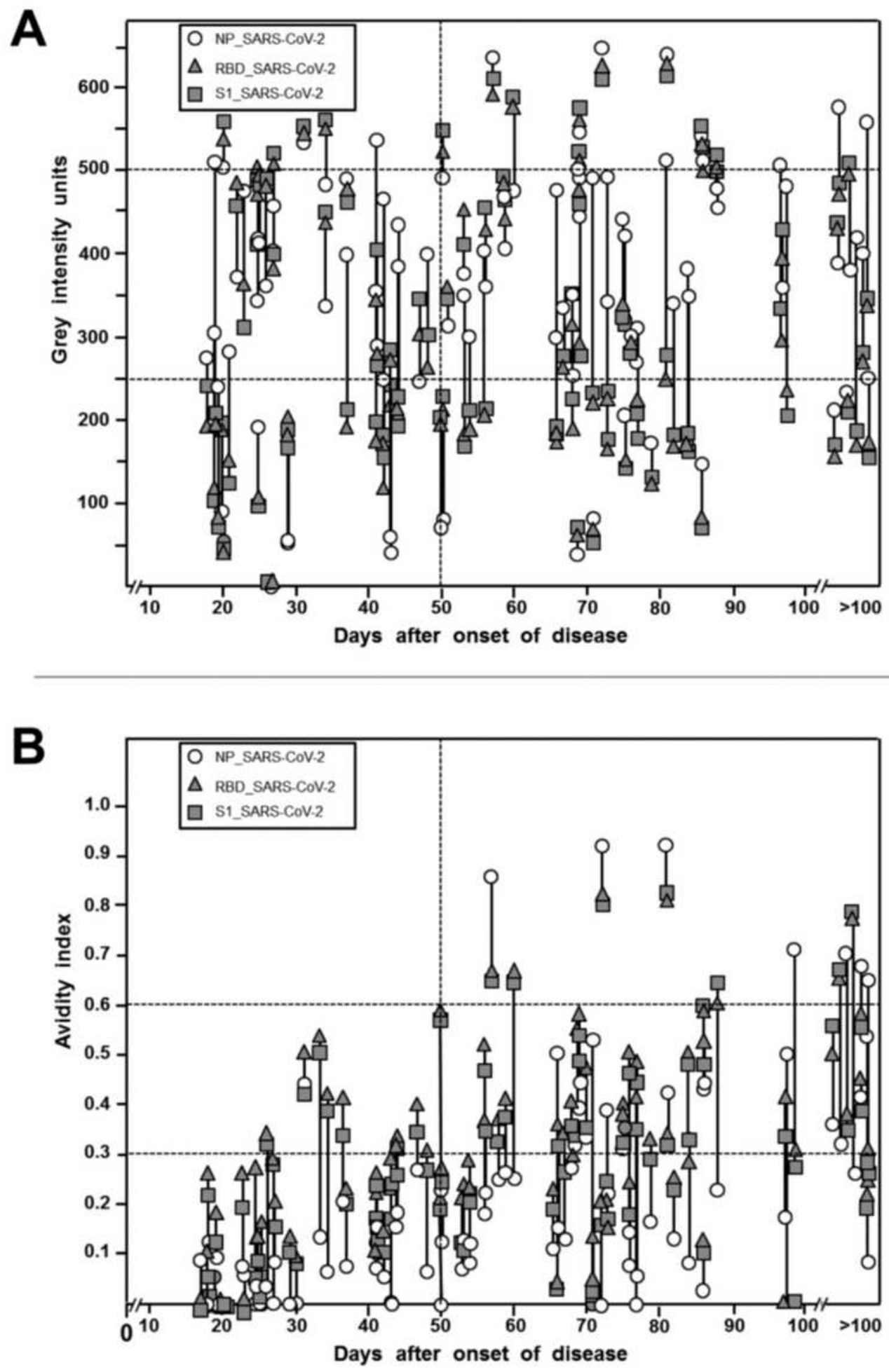

Figure 2

IgG responses in 93 serum samples from patients with confirmed SARS CoV-2 infection and COVID-19. 93 sera from patients with clinical signs of Covid-19, quite predominantly showing ambulatory mild disease, and with positive SARS CoV-2 PCR test were tested with the recomline SARS-CoV-2 IgG test. The grey intensities $(A)$, which reflect the concentrations of the respective antibodies and the avidity indices (B) are shown in correlation to the time after onset of disease for each serum. A. Reflectometric grey 
intensity values. The result shows a broad distribution of grey scales, which was similar at all time points. The percentages of sera showing values in the ranges 0-250, 250-500 and more than 500 grey intensity were not significantly different when sera taken before or after 50 days after onset of disease were compared, with the exception of IgG towards NP at less than 250 units. Importantly, all sera that gave a positive result showed positivity towards all three antigens tested, without exception. In $93.5 \%$ of the sera with positive IgG towards $S 1, \lg G$ towards RBD showed a value that was less than 20 grey intensity units different from that obtained for IgG $S 1$, whereas in $6.5 \%$ of the sera, IgG values towards $S 1$ and RBD were different more than 20 grey intensity units $(p<0.001)$. In contrast, only $15 \%$ of sera showed IgG towards $\mathrm{NP}$ at a value that differed less than 20 grey intensity units from that of the respective IgG S1 value and $84.9 \%$ showed higher difference $(p<0.001)$. B. Avidity indices. Whereas the grey intensity values (indicative of the respective concentrations of $\mathrm{IgG}$ ) were broadly similar between sera taken within 50 days or after 50 days after onset of disease, the avidity of the IgG was increasing between these groups. However, it did not reach complete avidity maturation (as seen for a reference virus under Supplementary materials). 82.9 percent of the sera taken before day 50 showed an avidity index below 0.3 , whereas only $42.7 \%$ of the sera taken after 50 days showed similar low avidity $(p<0.001)$. As an indication of partial avidity maturation, only $17.1 \%$ of sera taken before 50 days showed an avidity index between 0.3 and 0.6 and none an index above 0.6 , whereas $44 \%$ of the group taken after 50 days showed avidity indices between 0.3 and 0.6 , and $13.2 \%$ an index above 0.6 (indicative for nearly completed maturation) ( $p<$ 0.001). In summary, an overall low degree of avidity maturation was seen, with a minority of samples of high avidity at late time points. 

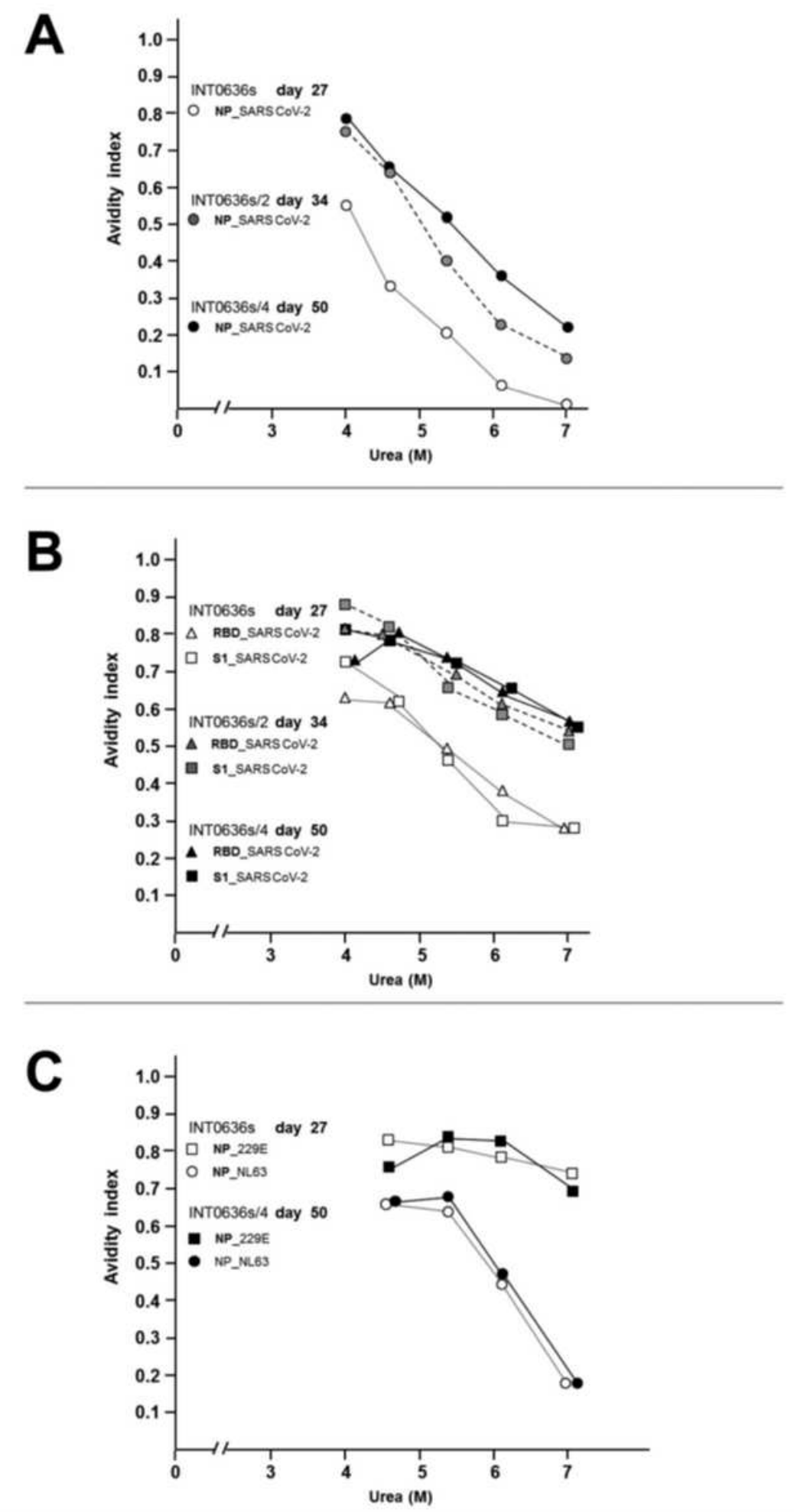

\section{Figure 3}

Demonstration of the abrogation of avidity maturation. Sera were available from a patient with Covid-19 and PCR-confirmed SARS CoV-2 infection taken at days 27, 34 and 50 after onset of disease. The sera were tested in avidity assays based on the recomLine SARS CoV-2 assay, with the modification that they remained either untreated with urea or were treated with the indicated concentrations of urea. This allows to titrate nominal avidity indices in relationship to the urea concentration. The shift of the resultant 
titration curves is indicative for avidity maturation. The figure shows that avidity for lgG towards NP (A) and RBD or S1 (B) had increased between day 27 and 34 ( $\mathrm{dt}=7$ days), but only showed minor (A) or no (B) further increase between days 34 and $50 \mathrm{dt}=16$ days). This finding is indicative for an abrogation of avidity maturation. Though the avidity indices for IgG towards RBD and S1 were higher compared to IgG towards NP, in none of the markers high avidity above 0.6 was reached. Remarkably, the avidity index of IgG towards the seasonal corona viruses 229E and NL63 either remained unchanged high (229E) or low (NL63) during the period of observation. This finding indicates that an incomplete avidity maturation can also be found for seasonal corona viruses. 

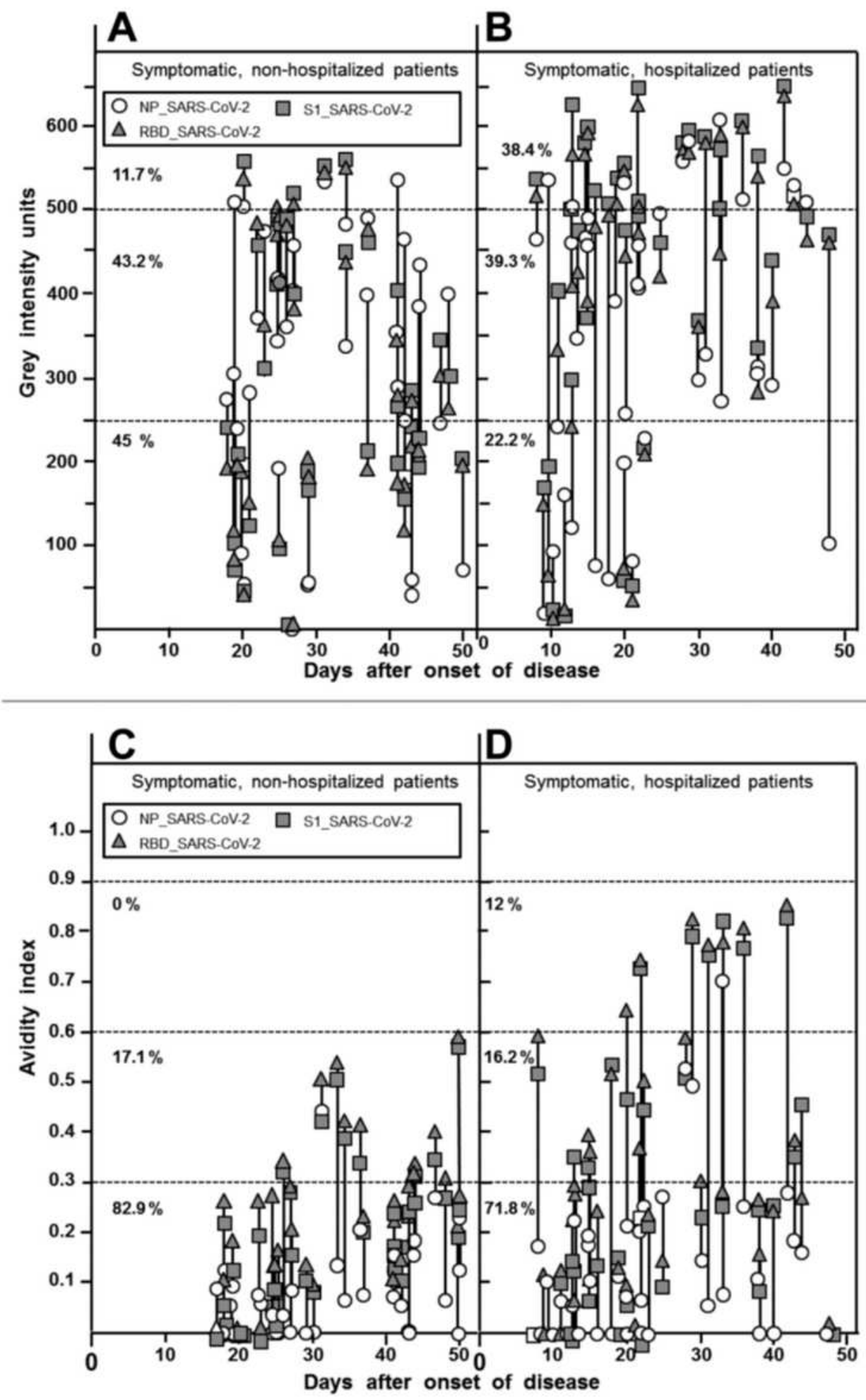

Figure 4

The serological response towards SARS CoV-2 in Covid-19 patients without and with the need for hospitalization. Sera from 37 patients with Covid-19 without need for hospitalization and 39 patients with need for hospitalization were taken within the first 50 days after onset of disease and tested in the recomLine SARS CoV-2 avidity assay. A. Optical density. Relatively less hospitalized patients with moderate or severe disease showed grey intensity values in the OD range from $0-250$ grey intensity 
units compared to nonhospitalized patients showing ambulatory mild disease ( $22.2 \%$ compared to $45 \%$; $\mathrm{p}<0.001)$ and relatively more hospitalized patients showed grey intensity values in the range larger than 500 units $(38.4 \%$ compared to $11.7 \%$; $<0.001)$, whereas there was no significant difference in the intermediate grey intensity range between 250 and 500 units $(39.3 \%$ compared to $43.2 \% ; p=0.6)$. B. Avidity indices. The percentages of sera with avidity indices between 0 and $0.3(71.8 \%$ versus $82.9 \%$ for hospitalized versus nonhospitalized patients), and 0.3-06 (16.2\% versus $17.1 \%$ for hospitalized versus nonhospitalized patients) were not significantly different. However, a certain percentage of hospitalized patients reached avidity indices $>0.6(12 \%)$, whereas nonhospitalized patients did not reach these high avidity indices $(p<0.001)$. These data show that hospitalized patients reach a substantial increase in antibody concentration (particularly after the first 25 days after onset of disease), whereas the increase in avidity is less substantial, though detectable with significance. 